\title{
The Effects of Ripening and Cold Storage on the Volatile Profiles of Three Japanese Plum Cultivars (Prunus salicina Lindl.) and One Interspecific Plum-apricot Cultivar
}

\author{
Esmé D. Louw ${ }^{1} \&$ Karen I. Theron ${ }^{1}$ \\ ${ }^{1}$ Department of Horticultural Science, Stellenbosch University, Stellenbosch, South Africa \\ Correspondence: Esmé Louw, Department of Horticultural Science, Stellenbosch University, Stellenbosch, \\ South Africa. Tel: 27-21-808-4760. E-mail: esmelouw@sun.ac.za
}

Received: July 6, 2012

doi:10.5539/jas.v4n11p257
Accepted: July 23, 2012

Online Published: October 16, 2012

\begin{abstract}
Volatile compounds contribute greatly to the flavour of fruit and can be altered by maturity and cold storage. The volatiles of three Japanese plum cultivars ('Sapphire', 'Songold', and 'Larry Anne') and one plumcot ('Flavor King') were determined at three functional stages: commercial harvest, tree-ripened fruit and cold stored fruit. HS-SPME was used for extraction and GC-TOFMS for separation and identification. A total of 52 compounds was found and classified into three groups ('unique' (28), 'generic' (10) and 'frequent' (14)) based on frequency of occurrence. Discriminant analysis showed that the volatile profiles of the three functional stages were distinct within each cultivar, and the main contributors to the patterns were identified. The volatile profiles of 'Larry Anne' and 'Flavor King' were the most affected by cold storage conditions. Inter-cultivar analysis indicated that the cultivars have different volatile profiles at each of the functional groups with 'Larry Anne' and 'Flavor King' showing the largest differences. 'Flavor King', a plumcot, presented a ripe volatile profile that was much diverged from that of the true plums.
\end{abstract}

Keywords: volatile compounds, plums, SPME, GC-TOF MS, cold storage

\section{Introduction}

Plums are classified as stone fruit within the genus Prunus, family Rosacea and are produced and cultivated globally for its palatability and unique aromatic characteristics. Other popular stone fruits within this genus include peaches, apricots, nectarines and cherries. Taste and flavour combine to create an eating experience whereby non-volatiles (sugars and acids) mainly contribute to taste and volatile compounds to flavour (Williams \& Ismail, 1981). In plums, as in most stone fruit, the physical (firmness, weight and appearance) and non-volatile composition had been documented well and are commercially used as indicators for optimum harvest dates and to define export grades. In contrast to this, the flavour components (volatiles) responsible for the distinct plum aroma, particularly in Japanese plums, are not as widely researched compared to apricots, peaches and nectarines.

The production of aroma volatiles during fruit maturation and ripening is dynamic and the pattern of volatile constituents, both qualitative and quantitative, can vary greatly (Agozzino, Avellone, Filizzola, Farina, \& Lo Bianco, 2007). The effects of prolonged exposure to low temperatures, i.e. cold storage on the volatile profile of stone fruit had been identified and documented for apricots (Aubert, Bony, Chalot, \& Hero, 2010) and peaches (Raffo, Nardo, Tabilio, \& Paoletti, 2008), but to date, no literature, apart from our research (Louw \& Theron, 2012) could be found for plums.

In South Africa, $78 \%$ of the total plum production (2011) is exported resulting in over 9 million cartons $(5.25 \mathrm{~kg}$ equivalent cartons) comprising of 35 different cultivars (PPECB Information portal: http://info.ppecb.com). Plums are harvested relatively unripe and ripen in cold storage while in transit (for up to 42 days) from farm to market. Prone to physiological disorders such as chilling injury and gel breakdown associated with cold storage, plums are shipped under a dual temperature regime. The fruit are first stored at $-0.5^{\circ} \mathrm{C}$ for 8 to 10 days followed by an increase in temperature to $7.5^{\circ} \mathrm{C}$ for a further 5 to 7 days after which the temperature is decreased back to $-0.5^{\circ} \mathrm{C}$ for up to 25 days. This protocol is flexible as some cultivars are more susceptible to the disorders than others. As plum consumers have indicated that their preference has now shifted towards flavour and taste 
(SASPA/Richmond Towers, \& UK consumer research de-brief, 2006) it is important to analyse the dynamics of the volatile profiles in early harvested plums exposed to cold storage to ensure that acceptable flavour is persevered throughout the marketing process.

Inter-specific hybrids between Japanese plums and apricots ( $P$. salicinax P. armeniaca) are commonly known as plumcots and are bred for their sweet taste and complex but excellent flavour (Blažek, 2007). 'Flavor King' is such a plumcot that has become a valued cultivar in South Africa over the last decade.

The aim of this study was to investigate the volatile profiles of three Japanese plum cultivars ('Sapphire', 'Songold', and 'Larry Anne') and one plumcot ('Flavor King') at three different functional stages: harvest (commercial harvest intended for export), fruit left to ripen on the tree and fruit exposed to the commercial cold storage regimes used in South Africa. Inter-and intra-cultivar comparisons using discriminate analysis were presented to describe any shifts in the volatile profiles caused by ripening and cold storage.

\section{Method}

\subsection{Fruit Selection, Harvesting and Storage Conditions}

Fruit from three commercial plum (Prunus salicina Lindl.) cultivars and one plumcot grown near Stellenbosch (Western Cape, South Africa) were used in this study. The cultivars (in order of ripening and harvest) were 'Sapphire', 'Flavor King' (plumcot), 'Songold' and 'Larry Anne'. Fruits were collected at two different picking dates from commercial orchards, trained to a flat trellis system. The first picking date coincided with the commercial harvest when fruit flesh firmness was close to the average parameters as stated by the South African governmental export regulations (Table 1). At this picking date, we identified and tagged ten trees that were not harvested. These trees were left to bear their fruit until ripe and ready to eat at a flesh firmness of $\pm 1-3 \mathrm{~kg}$. Fruit of similar size and colour were selected from the middle of the canopy approximately $1.5 \mathrm{~m}$ from the orchard floor. Fruits were transported to our laboratory, stored at ambient temperature and processed within 12 hours of harvest. A minimum of six fruit from both picking dates was processed to determine quality parameters and volatile profiles. A further 80 fruit from the first picking date were packed and cold-stored for up to 42 days according to commercial export protocols (Table 1). After storage, the fruits were transferred to $15^{\circ} \mathrm{C}$ and left to ripen to a flesh firmness of $\pm 1-3 \mathrm{~kg}$ after which a minimum of six fruits per cultivar was processed to determine quality parameters and volatile profiles. The study was conducted over two plum seasons (2008 \& 2009).

Table 1. Commercial harvest and storage conditions of the three plum and one plumcot cultivars

\begin{tabular}{|c|c|c|c|c|}
\hline Commercial harvest and storage conditions & 'Sapphire' & 'Songold' & 'Larry Anne' & 'Flavor King' (plumcot) \\
\hline Required firmness range at harvest & $9.50-5.00 \mathrm{~kg}$ & $\begin{array}{l}9.00-5.00 \mathrm{~kg} \\
(4.50 \mathrm{~kg} \text { if } 13 \% \mathrm{TSS})\end{array}$ & $9.00-5.50 \mathrm{~kg}$ & $8.50-5.50 \mathrm{~kg}$ \\
\hline Actual mean firmness at commercial harvest ( \pm stddev) & $6.81( \pm 1.10) \mathrm{kg}$ & $7.06( \pm 0.80) \mathrm{kg}$ & $7.27( \pm 1.01) \mathrm{kg}$ & $7.35( \pm 0.70) \mathrm{kg}$ \\
\hline Total cold storage time (days) & $42 \mathrm{~d}$ & $42 \mathrm{~d}$ & $42 \mathrm{~d}$ & $42 \mathrm{~d}$ \\
\hline \multirow[t]{3}{*}{ Cold storage protocol } & $10 \mathrm{~d} @-0.5^{\circ} \mathrm{C}$ & $10 \mathrm{~d} @-0.5^{\circ} \mathrm{C}$ & $10 \mathrm{~d} @-0.5^{\circ} \mathrm{C}$ & $10 \mathrm{~d} @-0.5^{\circ} \mathrm{C}$ \\
\hline & $7 \mathrm{~d} @ 7.5^{\circ} \mathrm{C}$ & $7 \mathrm{~d} @ 7.5^{\circ} \mathrm{C}$ & $7 \mathrm{~d} @ 7.5^{\circ} \mathrm{C}$ & $7 \mathrm{~d} @ 7.5^{\circ} \mathrm{C}$ \\
\hline & $25 \mathrm{~d} @-0.5^{\circ} \mathrm{C}$ & $25 \mathrm{~d} @-0.5^{\circ} \mathrm{C}$ & $25 \mathrm{~d} @-0.5^{\circ} \mathrm{C}$ & $25 \mathrm{~d} @-0.5^{\circ} \mathrm{C}$ \\
\hline
\end{tabular}

\subsection{Determination of Fruit Quality Parameters}

Material and methods in this section are similar to that used by Louw and Theron (2012). The range, mean and standard deviation values were determined for each quality parameter and are presented in Table 2.

\subsection{Volatile Sample Preparation, HS-SPME-GCTOF-MS Conditions and Identification}

Material and methods in this section are similar to that used by Louw and Theron (2012).

\subsection{Data Classifications and Statistical Analysis}

Fruit firmness was the maturity index used to create three functional groups namely, 'Harvest', 'Tree-ripened' and 'Stored'. The 'Harvest' group was represented by fruit picked and processed at commercial harvest when the average firmness was well within the export window of requirements (Table 1). These fruits are considered physiologically mature but due to "early" South African plum harvest practices are not "ripe-and-ready-to-eat". The 'Tree-ripened' group contained fruit left on the tree to ripen and picked and processed when considered "ripe-and-ready-to-eat" at a flesh firmness of $\pm 1-3 \mathrm{~kg}$. The 'Stored' groups were fruit picked during the commercial harvest and then stored and ripened (firmness of $\pm 1-3 \mathrm{~kg}$ ) according to commercial export protocols. Volatile data were subjected to a one-way analysis of variance (ANOVA) and comparison testing was done on the mean values of each compound using Fisher's LSD (The Least Significant Difference) test ( $p=0.05$ level). 
Discriminant Analysis (DA) was performed on the three functional groups within each cultivar and the forward stepwise method applied to identify volatiles that contribute significantly to the separation patterns. Inter-cultivar DA was performed for all the functional groups to compare the four cultivars and possibly identify sensitivity to commercial cold storage. Reclassification was done to validate the models. All calculations and modeling were performed using XLSTAT Version 2010.4.01.

Table 2. Mean ( \pm stddev) of the quality parameters determined for the three plum and one plumcot cultivars

\begin{tabular}{|c|c|c|c|c|c|c|c|c|c|c|c|c|}
\hline \multirow{3}{*}{ Non-volatile parameters } & \multicolumn{3}{|c|}{ 'Sapphire' } & \multicolumn{3}{|c|}{ 'Songold' } & \multicolumn{3}{|c|}{ 'Larry Anne' } & \multicolumn{3}{|c|}{ 'Flavor King' (plumcot) } \\
\hline & $\mathrm{H}$ & TR & $\mathrm{S}$ & $\mathrm{H}$ & TR & $\mathrm{S}$ & $\mathrm{H}$ & TR & $\mathrm{S}$ & $\mathrm{H}$ & TR & $\mathrm{S}$ \\
\hline & $\mathrm{n}=12$ & $\mathrm{n}=12$ & $\mathrm{n}=12$ & $\mathrm{n}=12$ & $\mathrm{n}=12$ & $\mathrm{n}=12$ & $\mathrm{n}=12$ & $\mathrm{n}=12$ & $\mathrm{n}=12$ & $\mathrm{n}=12$ & $\mathrm{n}=12$ & $\mathrm{n}=12$ \\
\hline \multirow{2}{*}{ Firmness (kg) } & 6.81 & 1.93 & 1.58 & 7.06 & 2.93 & 1.56 & 7.43 & 2.04 & 1.52 & 7.35 & 2.23 & 1.73 \\
\hline & $( \pm 1.1)$ & $( \pm 0.43)$ & $( \pm 0.36)$ & $( \pm 0.72)$ & $( \pm 0.39)$ & $( \pm 0.25)$ & $( \pm 1.01)$ & $( \pm 1.23)$ & $( \pm 0.29)$ & $( \pm 0.70)$ & $( \pm 1.91)$ & $( \pm 0.29)$ \\
\hline \multirow{2}{*}{ Weight (g) } & 102.75 & 82.58 & 88.17 & 106.83 & 129.00 & 95.33 & 111.08 & 103.25 & 80.00 & 102.50 & 90.67 & 99.08 \\
\hline & $( \pm 19.72)$ & $( \pm 17.20)$ & $( \pm 16.55)$ & $( \pm 15.23)$ & $( \pm 13.11)$ & $( \pm 10.35)$ & $( \pm 30.79)$ & $( \pm 35.53)$ & $( \pm 7.75)$ & $( \pm 9.22)$ & $( \pm 6.88)$ & $( \pm 16.19)$ \\
\hline \multirow{2}{*}{ Sugar (\% Brix) } & 11.39 & 12.97 & 12.53 & 11.31 & 14.94 & 11.85 & 16.14 & 16.52 & 13.97 & 15.36 & 17.59 & 14.38 \\
\hline & $( \pm 1.03)$ & $( \pm 1.85)$ & $( \pm 1.17)$ & $( \pm 0.60)$ & $( \pm 2.53)$ & $( \pm 1.09)$ & $( \pm 1.83)$ & $( \pm 2.49)$ & $( \pm 1.04)$ & $( \pm 0.81)$ & $( \pm 2.33)$ & $( \pm 1.45)$ \\
\hline \multirow{2}{*}{ Acid (\% malic acid) } & 1.50 & 1.10 & 1.24 & 1.62 & 1.22 & 1.20 & 1.82 & 1.00 & 1.28 & 1.30 & 0.85 & 0.91 \\
\hline & $( \pm 0.17)$ & $( \pm 0.19)$ & $( \pm 0.18)$ & $( \pm 0.13)$ & $( \pm 0.09)$ & $( \pm 0.15)$ & $( \pm 0.18)$ & $( \pm 0.23)$ & $( \pm 0.38)$ & $( \pm 0.20)$ & $( \pm 0.28)$ & $( \pm 0.11)$ \\
\hline \multirow{2}{*}{ Sugar:Acid } & 7.70 & 11.93 & 10.32 & 7.01 & 12.18 & 10.12 & 8.89 & 17.53 & 11.49 & 12.23 & 22.51 & 15.96 \\
\hline & $( \pm 1.01)$ & $( \pm 1.77)$ & $( \pm 1.56)$ & $( \pm 0.64)$ & $( \pm 1.59)$ & $( \pm 2.29)$ & $( \pm 0.59)$ & $( \pm 5.86)$ & $( \pm 2.31)$ & $( \pm 2.95)$ & $( \pm 6.63)$ & $( \pm 2.64)$ \\
\hline
\end{tabular}

$\mathrm{H}=$ 'Harvest', $\mathrm{TR}=$ 'Tree-ripened', $\mathrm{S}=$ 'Stored' functional groups.

\section{Results}

\subsection{Quality Parameters}

The other quality parameters, apart from firmness, showed obvious differences when comparing the functional groups (Table 2). These include slight weight loss during storage and increased TSS during tree ripening as would be expected. Average fruit weight of the 'Stored' group was always found to be less than in the 'Harvest' group for all the cultivars. Similarly, the sugar (TSS) levels of the 'Stored' fruits were lower than those of the 'Harvest' fruit due to the fruits' inability to replenish the carbohydrates metabolised during biological processes once separated from the tree. 'Tree-ripened' fruits were often bigger in size and weight and had higher sugar content when compared to 'Harvest' and 'Stored' fruit possibly due to a longer opportunity to develop while attached to the tree during ripening. The opposite pattern was observed for acid levels in the 'Tree-ripened' and 'Stored' samples. This is expected as it is well known that acid levels drop during the ripening process to produce a more palatable fruit. The cultivars with the highest sugar levels were samples from 'Flavor King' and 'Larry Anne' fruit in the 'Tree-ripened' group with levels as high as 19.0\% Brix ('Larry Anne') and 21.0\% Brix ('Flavor King'). The lowest average acid level was also present in the 'Tree-ripened' samples of 'Flavor King' $(0.85( \pm 0.28) \%$ malic acid $)$ and 'Larry Anne' $(1.00( \pm 0.23) \%$ malic acid $)$, resulting in relatively high sugar-to-acid ratios for these two cultivars.

\subsection{General Trends in the Volatile Profiles}

A total of 52 volatile compounds was measured for all four cultivars, and mean values are presented in Table 3 . As the current study used identical methods to that presented by Louw and Theron (2012) we can discuss the results in tandem, subsequently increasing the number of cultivars under review to seven, and the total number of volatiles detected to 62 (Table 4). Of the 52 compounds found in the present study, 28 (54\%) were only present in one of the four cultivars compared to $31(50 \%)$ of the 62 compounds present in the seven cultivars when combining the data with that found by Louw and Theron (2012) (Table 4). We classified these compounds as 'unique' to the cultivar, although we in no way suggest that it is not present in other plum cultivars not included in the two studies or at levels below our detection range. Due to the low frequency of occurrence, it is likely that these components contribute to the cultivar specific aroma of plums rather than the general plum aroma. 
Of the remaining 24 compounds in the present study, the following 10 compounds were measured in all four of the cultivars: hexanal, 2-hexenal, trans linalool oxide, cis linalool oxide, linalool, $\alpha$-terpineol, p-menth-1-en-9-al, $\beta$-damascenone, $2 \mathrm{H}$-pyran,3,6.dihydro-4-methyl-2-(2-methyl-1-propenyl) and $\beta$-ionone. Combining the results with Louw and Theron (2012) we found only eight compounds present in all seven the cultivars. The reduction was caused by $2 \mathrm{H}$-pyran,3,6.dihydro-4-methyl-2-(2-methyl-1-propenyl) not detected in 'Laetitia' nor 'Angeleno' and $\beta$-ionone not present in 'Angeleno' (Louw \& Theron, 2012). As a result of the high frequency of occurrence of these eight compounds amongst the seven cultivars we classified them as 'generic' (Table 4) and suggest that they are more likely to contribute to the general plum aroma.

The remaining 14 compounds of the present study were shared amongst two or three of the four cultivars and classified as 'frequent', implying that they are often found in plum cultivars. When the results from Louw and Theron (2012) are included the number increases to 23 as a number of compounds initially thought to be unique are now shared with the three cultivars from Louw and Theron (Table 4).

Table 3. Chemical groups and means of the volatiles found in 'Sapphire', 'Songold', 'Larry Anne' and 'Flavor King'

\begin{tabular}{|c|c|c|c|c|c|c|c|c|c|c|c|c|c|c|}
\hline \multirow{2}{*}{$\begin{array}{l}\text { Chemical group } \\
\text { and RT order }\end{array}$} & \multirow{2}{*}{$\mathrm{Abb}$} & \multirow{2}{*}{ Aroma compounds } & \multicolumn{2}{|c|}{ 'Sapphire' } & \multicolumn{4}{|c|}{ 'Flavor King' } & \multicolumn{2}{|l|}{ 'Songold' } & \multicolumn{4}{|c|}{ 'Lany Anne' } \\
\hline & & & $\mathrm{H}$ & TR & $\mathrm{s}$ & $\mathrm{H}$ & TR & S & $\mathrm{H}$ & TR & $\mathrm{S}$ & $\mathrm{H}$ & TR & S \\
\hline \multicolumn{15}{|l|}{ Acids } \\
\hline 38 & Nona & Nonanoic acid* & $0.094 \mathrm{a}$ & $0.082 \mathrm{a}$ & $0.052 \mathrm{a}$ & $0.024 \mathrm{~b}$ & $\mathrm{NDa}$ & $\mathrm{NDa}$ & ND & ND & ND & ND & ND & $\mathrm{ND}$ \\
\hline 46 & Eug & Eugenolmethylether* & $0.002 \mathrm{a}$ & $0.091 \mathrm{a}$ & $0.033 \mathrm{a}$ & $\mathrm{ND}$ & $\mathrm{ND}$ & ND & $\mathrm{ND}$ & $\mathrm{ND}$ & $\mathrm{ND}$ & $\mathrm{ND}$ & $\mathrm{ND}$ & $\mathrm{ND}$ \\
\hline \multicolumn{15}{|l|}{ Alcohols } \\
\hline 4 & Hex & 1-Hexanol** & ND & $\mathrm{ND}$ & ND & ND & ND & ND & $0.008 \mathrm{a}$ & $0.184 \mathrm{~b}$ & $0.422 \mathrm{c}$ & ND & ND & $\mathrm{ND}$ \\
\hline 8 & 3Hexe & cis-3-Hexenol* & ND & $\mathrm{ND}$ & ND & $\mathrm{NDa}$ & $0.102 \mathrm{a}$ & $1.081 \mathrm{~b}$ & ND & ND & ND & ND & ND & $\mathrm{ND}$ \\
\hline 30 & cMenth & cis-p-Menth-2,8-dienol* & ND & $\mathrm{ND}$ & ND & ND & ND & ND & $\mathrm{ND}$ & ND & ND & $0.218 b$ & $0.135 \mathrm{a}$ & $0.122 \mathrm{a}$ \\
\hline 32 & Cymol & p-Cymen-8-ol* & ND & $\mathrm{ND}$ & ND & $\mathrm{ND}$ & ND & ND & $0.003 \mathrm{~b}$ & $0.001 \mathrm{ab}$ & $\mathrm{NDa}$ & $\mathrm{ND}$ & $\mathrm{ND}$ & $\mathrm{ND}$ \\
\hline \multicolumn{15}{|l|}{ Aldehydes } \\
\hline 1 & Hexa & Hexanal** & $0.660 \mathrm{a}$ & $1.095 \mathrm{a}$ & $0.588 \mathrm{a}$ & $0.762 b$ & $0.240 \mathrm{a}$ & $0.086 \mathrm{a}$ & $0.205 b$ & $0.265 \mathrm{~b}$ & $0.065 \mathrm{a}$ & $0.723 \mathrm{a}$ & $1.360 \mathrm{~b}$ & $1.053 \mathrm{ab}$ \\
\hline 3 & Hexe & 2-Hexenal** & $1.910 \mathrm{a}$ & $1.777 \mathrm{a}$ & $4.603 \mathrm{~b}$ & $0.771 \mathrm{~b}$ & $\mathrm{NDa}$ & $\mathrm{NDa}$ & $1.253 \mathrm{a}$ & $1.286 \mathrm{a}$ & $0.808 \mathrm{a}$ & $2.030 \mathrm{a}$ & $2.574 \mathrm{a}$ & $3.669 \mathrm{~b}$ \\
\hline 5 & Non & Nonanal* & $0.620 \mathrm{a}$ & $0.651 \mathrm{a}$ & $1.030 \mathrm{a}$ & $\mathrm{ND}$ & ND & ND & ND & ND & ND & $\mathrm{ND}$ & ND & $\mathrm{ND}$ \\
\hline 11 & Benz & Benzaldehyde** & $\mathrm{ND}$ & $\mathrm{ND}$ & ND & $0.038 \mathrm{a}$ & $0.036 \mathrm{a}$ & $0.032 \mathrm{a}$ & $0.009 \mathrm{ab}$ & $0.012 \mathrm{~b}$ & $0.002 \mathrm{a}$ & $0.018 \mathrm{a}$ & $0.015 \mathrm{a}$ & $0.055 \mathrm{~b}$ \\
\hline 29 & Iso & $\begin{array}{l}\text { 2-Isopropylidene- } \\
\text { 5-methylhex-4enal* }\end{array}$ & $0.041 \mathrm{a}$ & $0.220 \mathrm{~b}$ & $0.056 \mathrm{a}$ & ND & ND & $\mathrm{ND}$ & ND & ND & ND & ND & ND & $\mathrm{ND}$ \\
\hline 36 & pMenth & p-Menth-1-en-9-al* & $0.098 \mathrm{a}$ & $0.093 \mathrm{a}$ & $0.251 \mathrm{a}$ & $0.469 \mathrm{a}$ & $0.989 \mathrm{~b}$ & $0.598 \mathrm{a}$ & $0.146 \mathrm{~b}$ & $0.052 \mathrm{a}$ & $0.094 \mathrm{ab}$ & $0.705 \mathrm{c}$ & $0.374 a$ & $0.548 \mathrm{~b}$ \\
\hline \multicolumn{15}{|l|}{ Esters } \\
\hline 2 & But & Butyl acetate* & ND & $\mathrm{ND}$ & ND & $2.866 \mathrm{a}$ & $17.700 \mathrm{a}$ & $52.685 \mathrm{~b}$ & ND & ND & ND & ND & ND & ND \\
\hline 9 & Hexfor & n-Hexylformate* & ND & $\mathrm{ND}$ & ND & $0.224 \mathrm{a}$ & $1.418 \mathrm{~b}$ & $0.543 \mathrm{a}$ & ND & ND & ND & ND & ND & ND \\
\hline 10 & Amyl & Amylacetate* & $\mathrm{ND}$ & $\mathrm{ND}$ & $\mathrm{ND}$ & $0.183 \mathrm{a}$ & $0.284 \mathrm{a}$ & $0.631 \mathrm{~b}$ & $\mathrm{ND}$ & $\mathrm{ND}$ & ND & ND & ND & $\mathrm{ND}$ \\
\hline 12 & Hexyl & $\mathrm{n}$-Hexyl acetate* & $\mathrm{NDa}$ & $0.385 \mathrm{~b}$ & $0.221 a b$ & $4.529 \mathrm{a}$ & $25.412 \mathrm{~b}$ & $13.922 \mathrm{ab}$ & ND & ND & ND & $\mathrm{NDa}$ & $0.471 \mathrm{~b}$ & $0.120 \mathrm{a}$ \\
\hline 17 & Butbu & Butylbutanoate* & ND & $\mathrm{ND}$ & ND & $0.009 \mathrm{a}$ & $0.060 \mathrm{a}$ & $0.457 \mathrm{~b}$ & ND & ND & ND & ND & ND & $\mathrm{ND}$ \\
\hline 18 & 3Hexac & cis-3-Hexenyl acetate* & ND & $\mathrm{ND}$ & ND & $0.275 \mathrm{a}$ & $0.406 \mathrm{a}$ & $1.028 \mathrm{~b}$ & ND & ND & ND & ND & ND & ND \\
\hline 27 & Нер & Heptyl acetate* & ND & $\mathrm{ND}$ & ND & $0.019 \mathrm{a}$ & $0.123 \mathrm{~b}$ & $0.012 \mathrm{a}$ & ND & $\mathrm{ND}$ & $\mathrm{ND}$ & ND & $\mathrm{ND}$ & $\mathrm{ND}$ \\
\hline 34 & Oct & Octyl acetate* & $\mathrm{ND}$ & $\mathrm{ND}$ & ND & $\mathrm{NDa}$ & $0.197 \mathrm{~b}$ & $0.017 \mathrm{a}$ & ND & ND & ND & ND & ND & $\mathrm{ND}$ \\
\hline 42 & Pent & Pentamethylene acetate* & ND & $\mathrm{ND}$ & ND & $\mathrm{NDa}$ & $0.090 \mathrm{~b}$ & $0.003 \mathrm{a}$ & ND & ND & ND & ND & ND & ND \\
\hline \multicolumn{15}{|l|}{ Furan } \\
\hline 7 & Eth & 2-Ethylfuran* & $\mathrm{ND}$ & $\mathrm{ND}$ & $\mathrm{ND}$ & $\mathrm{ND}$ & $\mathrm{ND}$ & ND & $0.065 \mathrm{a}$ & $0.162 \mathrm{~b}$ & $0.012 \mathrm{a}$ & $\mathrm{ND}$ & $\mathrm{ND}$ & $\mathrm{ND}$ \\
\hline 13 & Hexen & 2-Hexen--olide* & ND & $\mathrm{ND}$ & $\mathrm{ND}$ & $0.053 \mathrm{~b}$ & $\mathrm{NDa}$ & $\mathrm{NDa}$ & $0.053 \mathrm{a}$ & $0.199 \mathrm{~b}$ & $0.009 \mathrm{a}$ & $0.050 \mathrm{a}$ & $0.230 \mathrm{~b}$ & $0.053 \mathrm{a}$ \\
\hline 21 & tLinox & trans-Linalool oxide** & $0.079 \mathrm{a}$ & $0.048 \mathrm{a}$ & $0.084 \mathrm{a}$ & $0.769 b$ & $0.299 \mathrm{a}$ & $0.337 \mathrm{~b}$ & $0.185 b$ & $0.065 \mathrm{a}$ & $0.081 \mathrm{a}$ & $0.780 \mathrm{c}$ & $0.300 \mathrm{a}$ & $0.507 \mathrm{~b}$ \\
\hline 22 & cLinox & cis-Linalool oxide** & $0.057 \mathrm{a}$ & $0.034 \mathrm{a}$ & $0.032 \mathrm{a}$ & $0.256 \mathrm{~b}$ & $0.109 \mathrm{a}$ & $0.111 \mathrm{a}$ & $0.049 b$ & $0.009 \mathrm{a}$ & $0.022 \mathrm{a}$ & $0.329 \mathrm{c}$ & $0.132 \mathrm{a}$ & $0.182 \mathrm{~b}$ \\
\hline 28 & Pyran3 & $\begin{array}{l}\text { 2H-Pyran,3,6.dihydro-4methyl } \\
\text {-2-(2-methyl-1-propenyl)* }\end{array}$ & $0.130 \mathrm{a}$ & $0.065 \mathrm{a}$ & $0.064 \mathrm{a}$ & $0.108 \mathrm{~b}$ & $0.042 \mathrm{a}$ & $0.057 \mathrm{ab}$ & $0.036 \mathrm{~b}$ & $0.009 \mathrm{a}$ & $0.004 \mathrm{a}$ & $0.202 b$ & $0.037 \mathrm{a}$ & $0.054 \mathrm{a}$ \\
\hline \multicolumn{15}{|l|}{ Hydrocarbons } \\
\hline 24 & Hot & Hotrienol* & $0.400 \mathrm{a}$ & $0.495 \mathrm{a}$ & $0.721 \mathrm{a}$ & $0.799 \mathrm{~b}$ & $0.377 \mathrm{a}$ & $0.774 \mathrm{~b}$ & ND & ND & ND & $0.861 \mathrm{~b}$ & $0.535 \mathrm{a}$ & $0.619 \mathrm{a}$ \\
\hline 26 & Cyclo & $\begin{array}{l}1,3, \text { Cyclo-hexadiene } \\
1,3,5,5 \text {,tetra-methyl* }\end{array}$ & $0.021 \mathrm{a}$ & $0.031 \mathrm{a}$ & $0.020 \mathrm{a}$ & ND & ND & ND & ND & ND & ND & ND & ND & $\mathrm{ND}$ \\
\hline 37 & Bor & 2-Bomene* & $0.019 \mathrm{a}$ & $0.020 \mathrm{a}$ & $0.058 \mathrm{a}$ & $0.010 \mathrm{~b}$ & $0.008 \mathrm{ab}$ & $0.001 \mathrm{a}$ & ND & ND & ND & $0.030 \mathrm{~b}$ & $0.009 \mathrm{a}$ & $0.010 \mathrm{a}$ \\
\hline
\end{tabular}




\begin{tabular}{|c|c|c|c|c|c|c|c|c|c|c|c|c|c|c|}
\hline \multirow{2}{*}{$\begin{array}{l}\text { Chemical group } \\
\text { and RT order }\end{array}$} & \multirow{2}{*}{ Abb } & \multirow{2}{*}{ Aroma compounds } & \multicolumn{2}{|c|}{ 'Sapphire' } & \multicolumn{4}{|c|}{ 'Flavor King' } & \multicolumn{2}{|l|}{ 'Songold' } & \multicolumn{4}{|c|}{ 'Lary Anne' } \\
\hline & & & $\mathrm{H}$ & TR & $\mathrm{S}$ & $\mathrm{H}$ & TR & $\mathrm{S}$ & $\mathrm{H}$ & TR & $\mathrm{S}$ & $\mathrm{H}$ & TR & $\mathrm{S}$ \\
\hline 44 & 2But & $\begin{array}{c}2-[(2 \mathrm{E})-2 \text {-Butenyl] }-1,3,5- \\
\text { trimethylbenzene* }\end{array}$ & $\mathrm{ND}$ & $\mathrm{ND}$ & $\mathrm{ND}$ & $\mathrm{ND}$ & $\mathrm{ND}$ & $\mathrm{ND}$ & $\mathrm{ND}$ & ND & $\mathrm{ND}$ & $0.093 \mathrm{~b}$ & $0.083 \mathrm{~b}$ & $0.024 \mathrm{a}$ \\
\hline 47 & Trimeth & $\begin{array}{l}\text { 4-(2,4,4-Trimetyl-cyclohexa-1,5- } \\
\text { dienyl) but-3-en-2-one* }\end{array}$ & $0.021 \mathrm{a}$ & $0.034 \mathrm{a}$ & $0.176 \mathrm{~b}$ & $0.008 \mathrm{a}$ & $0.007 \mathrm{a}$ & $0.002 \mathrm{a}$ & $0.019 \mathrm{~b}$ & $0.002 \mathrm{a}$ & $0.016 \mathrm{~b}$ & ND & ND & ND \\
\hline 50 & Trimeth6 & $\begin{array}{l}\text { 4-(2,6,6-Trimethyl-cyclohexa- } \\
\text { 1,5,-diemethyl)but-3-en-2-one* }\end{array}$ & $0.021 \mathrm{a}$ & $0.029 \mathrm{a}$ & $0.087 \mathrm{~b}$ & $0.008 \mathrm{ab}$ & $0.009 \mathrm{~b}$ & $0.002 \mathrm{a}$ & $0.016 \mathrm{a}$ & $0.009 \mathrm{a}$ & $0.012 \mathrm{a}$ & ND & ND & ND \\
\hline \multicolumn{15}{|l|}{ Lactones } \\
\hline 49 & Deca & $\gamma$-Decalactone* & $0.011 \mathrm{a}$ & $0.173 \mathrm{a}$ & $0.537 \mathrm{~b}$ & $0.094 \mathrm{a}$ & $1.255 \mathrm{~b}$ & $0.010 \mathrm{a}$ & $\mathrm{ND}$ & $\mathrm{ND}$ & $\mathrm{ND}$ & $0.002 \mathrm{a}$ & $0.487 \mathrm{~b}$ & $0.435 \mathrm{~b}$ \\
\hline 52 & Dodeca & $\gamma$-Dodecalactone* & $0.004 a$ & $0.055 \mathrm{a}$ & $0.054 \mathrm{a}$ & $0.059 \mathrm{a}$ & $0.974 \mathrm{~b}$ & $0.012 \mathrm{a}$ & $\mathrm{ND}$ & ND & ND & $0.001 \mathrm{a}$ & $0.318 \mathrm{~b}$ & $0.095 \mathrm{a}$ \\
\hline \multicolumn{15}{|l|}{ Norisoprenoids } \\
\hline 39 & alon & $\alpha$-Ionone** & $\mathrm{ND}$ & ND & ND & $0.198 \mathrm{~b}$ & $0.109 \mathrm{~b}$ & $0.009 \mathrm{a}$ & $0.393 \mathrm{~b}$ & $0.169 \mathrm{a}$ & $0.080 \mathrm{a}$ & $0.019 \mathrm{c}$ & $0.011 \mathrm{~b}$ & $0.003 \mathrm{a}$ \\
\hline 43 & bDam & $\beta$-Damascenone** & $0.182 \mathrm{a}$ & $0.238 \mathrm{a}$ & $0.363 \mathrm{a}$ & $0.502 \mathrm{a}$ & $0.513 \mathrm{a}$ & $0.308 \mathrm{a}$ & $0.275 \mathrm{~b}$ & $0.280 \mathrm{~b}$ & $0.069 \mathrm{a}$ & $0.184 \mathrm{c}$ & $0.116 \mathrm{~b}$ & $0.066 \mathrm{a}$ \\
\hline 51 & blon & $\beta$-Ionone** & $0.024 \mathrm{a}$ & $0.036 \mathrm{a}$ & $0.052 \mathrm{a}$ & $0.012 \mathrm{a}$ & $0.028 \mathrm{~b}$ & $0.004 \mathrm{a}$ & $0.006 \mathrm{a}$ & $0.015 \mathrm{~b}$ & $0.008 \mathrm{ab}$ & $0.029 \mathrm{a}$ & $0.078 \mathrm{~b}$ & $0.013 \mathrm{a}$ \\
\hline \multicolumn{15}{|l|}{ Phenols } \\
\hline 14 & Phe & Phenol* & $\mathrm{ND}$ & $\mathrm{ND}$ & $\mathrm{ND}$ & ND & $\mathrm{ND}$ & $\mathrm{ND}$ & $0.077 \mathrm{~b}$ & $0.001 \mathrm{a}$ & $0.001 \mathrm{a}$ & $\mathrm{ND}$ & ND & $\mathrm{ND}$ \\
\hline \multicolumn{15}{|l|}{ Terpenes } \\
\hline 6 & Pin & $\alpha$-Pinene* & $0.039 \mathrm{a}$ & $0.079 \mathrm{~b}$ & $0.024 \mathrm{a}$ & $\mathrm{ND}$ & ND & $\mathrm{ND}$ & $\mathrm{ND}$ & ND & $\mathrm{ND}$ & ND & $\mathrm{ND}$ & $\mathrm{ND}$ \\
\hline 15 & Limox & Limonene oxide** & ND & $\mathrm{ND}$ & $\mathrm{ND}$ & $\mathrm{ND}$ & $\mathrm{ND}$ & $\mathrm{ND}$ & $\mathrm{ND}$ & ND & $\mathrm{ND}$ & $0.117 \mathrm{c}$ & $0.055 \mathrm{~b}$ & $0.032 \mathrm{a}$ \\
\hline 16 & Car & 3-Carene ** & $0.157 \mathrm{a}$ & $0.046 \mathrm{a}$ & $0.014 \mathrm{a}$ & $\mathrm{ND}$ & ND & $\mathrm{ND}$ & $\mathrm{ND}$ & ND & ND & $0.060 \mathrm{~b}$ & $0.011 \mathrm{a}$ & $0.011 \mathrm{a}$ \\
\hline 19 & Cym & $\beta$-Cymene** & $\mathrm{ND}$ & $\mathrm{ND}$ & $\mathrm{ND}$ & ND & ND & ND & $0.040 \mathrm{a}$ & $0.008 \mathrm{~b}$ & $0.006 \mathrm{~b}$ & $0.019 \mathrm{~b}$ & $0.003 \mathrm{~b}$ & $0.005 \mathrm{~b}$ \\
\hline 20 & Lim & D-Limonene** & $\mathrm{ND}$ & ND & $\mathrm{ND}$ & $\mathrm{ND}$ & ND & ND & ND & ND & ND & $0.053 \mathrm{~b}$ & $0.020 \mathrm{a}$ & $0.024 \mathrm{ab}$ \\
\hline 23 & Lina & Linalool** & $0.286 \mathrm{a}$ & $0.576 \mathrm{a}$ & $0.628 \mathrm{a}$ & $0.190 \mathrm{~b}$ & $0.152 \mathrm{~b}$ & $0.041 \mathrm{a}$ & $0.059 \mathrm{~b}$ & $0.013 \mathrm{a}$ & $0.025 \mathrm{a}$ & $0.688 \mathrm{~b}$ & $0.622 \mathrm{~b}$ & $0.337 \mathrm{c}$ \\
\hline 25 & Oci & Ocimenol* & $0.062 \mathrm{a}$ & $0.057 \mathrm{a}$ & $0.018 \mathrm{a}$ & $0.041 \mathrm{~b}$ & $0.044 \mathrm{~b}$ & $0.004 \mathrm{a}$ & $\mathrm{ND}$ & ND & ND & $0.169 b$ & $0.035 \mathrm{a}$ & $0.040 \mathrm{a}$ \\
\hline 33 & aTerpol & $\alpha$-Terpineol** & $0.206 \mathrm{a}$ & $0.235 \mathrm{a}$ & $0.477 \mathrm{a}$ & 0.191 a & $0.261 \mathrm{a}$ & $0.163 \mathrm{a}$ & $0.018 \mathrm{~b}$ & $0.006 \mathrm{a}$ & $0.007 \mathrm{~b}$ & $0.815 \mathrm{~b}$ & $0.277 \mathrm{a}$ & $0.273 \mathrm{a}$ \\
\hline 48 & Gera & cis-Geranylacetone* & $0.007 \mathrm{a}$ & $0.013 \mathrm{a}$ & $0.016 \mathrm{a}$ & $\mathrm{ND}$ & $\mathrm{ND}$ & $\mathrm{ND}$ & $\mathrm{ND}$ & $\mathrm{ND}$ & $\mathrm{ND}$ & $\mathrm{ND}$ & $\mathrm{ND}$ & $\mathrm{ND}$ \\
\hline \multicolumn{15}{|l|}{ Unidentified } \\
\hline 31 & U6 & Unidentified 6 & ND & ND & ND & $\mathrm{ND}$ & ND & $\mathrm{ND}$ & $0.055 \mathrm{ab}$ & $0.045 \mathrm{a}$ & $0.097 \mathrm{~b}$ & $\mathrm{ND}$ & $\mathrm{ND}$ & $\mathrm{ND}$ \\
\hline 35 & U9 & Unidentified 9 & ND & $\mathrm{ND}$ & ND & $\mathrm{ND}$ & ND & $\mathrm{ND}$ & $\mathrm{ND}$ & ND & $\mathrm{ND}$ & $0.096 \mathrm{~b}$ & $0.043 \mathrm{a}$ & $0.039 \mathrm{a}$ \\
\hline 40 & U7 & Unidentified 7 & ND & $\mathrm{ND}$ & $\mathrm{ND}$ & $\mathrm{ND}$ & ND & ND & $0.110 \mathrm{~b}$ & $0.057 \mathrm{a}$ & $0.052 \mathrm{a}$ & ND & $\mathrm{ND}$ & ND \\
\hline 41 & U8 & Unidentified 8 & ND & $\mathrm{ND}$ & $\mathrm{ND}$ & $0.052 \mathrm{a}$ & $0.040 \mathrm{a}$ & $0.069 \mathrm{a}$ & $\mathrm{ND}$ & $\mathrm{ND}$ & $\mathrm{ND}$ & $\mathrm{ND}$ & $\mathrm{ND}$ & ND \\
\hline 45 & U10 & Unidentified 10 & ND & ND & ND & ND & ND & ND & ND & ND & ND & $0.057 \mathrm{ab}$ & $0.078 \mathrm{~b}$ & $0.027 \mathrm{a}$ \\
\hline
\end{tabular}

The retention time (RT) order represents the chronological order in which the compounds were separated, starting with hexanal at $\pm 5.3 \mathrm{~min}$. and ending with $\gamma$-dodecalactone at $\pm 30.25 \mathrm{~min} . *$ indicates compounds identified via the NIST library and $* *$ indicates identification via NIST library and confirmation using commercial standards. $\mathrm{H}=$ 'Harvest', $\mathrm{TR}=$ 'Tree-ripened', $\mathrm{S}=$ 'Stored' functional groups. $\mathrm{ND}=$ not detected.Abb $=$ abbreviation. Different letters $(\mathrm{a}, \mathrm{b}$ and $\mathrm{c})$ indicate significant differences between values within each functional group according to the Fischer LSD test $(p=0.05)$.

\section{3 'Sapphire' Volatile Profile}

In 'Sapphire' samples, we measured a total of 26 different compounds (Table 4) which were present in all the groups except for n-hexyl acetate that was not detected in the 'Harvest' samples (Table 3). Of the 26 compounds, 18 showed no significant differences amongst any of the functional groups implying relative similar patterns in up to $70 \%$ of the volatile profile. Interestingly, 'Sapphire' contained six unique compounds including nonanal, $\alpha$-pinene, 1,3,cyclo-hexadiene1,3,5,5,tetra-methyl, eugenolmethylether, 2-isopropylidene-5-methylhex-4-enal and cis-geranylacetone (Table 4). These compounds account for $23 \%$ of the total number of 'Sapphire' compounds ranking second highest in a number of unique compounds, only surpassed by 'Flavor King' which is not a true plum, but a plum $\mathrm{x}$ apricot hybrid. Cold storage significantly affected only six of the volatile compounds in such a way that four of the six compounds (2-hexenal, 4-(2,6,6-trimethyl-cyclohexa-1,5,-diemethyl)but-3-en-2-one), $\quad \gamma$-decalactone and 4-(2,4,4-trimetyl-cyclohexa-1,5-dienyl)but-3-en-2-one) had drastically increased their levels with the latter increasing by up to five fold when compared to fruit ripened on the tree (Table 3). On the contrary, the levels of 2-isopropylidene-5-methylhex-4-enal and $\alpha$-pinene (two unique compounds) failed to rise when ripened after cold storage as in the case of tree-ripened fruit, but remained at the same relatively low levels as seen in the 
harvest fruit.

Table 4. Cultivars and aroma compounds classified according to frequency of occurrence

\begin{tabular}{|c|c|c|c|}
\hline \multirow{9}{*}{ 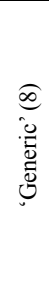 } & $\mathrm{Abb}$ & Aroma compound & Cultivars compound was detected in \\
\hline & Hexa & Hexanal & All \\
\hline & Hexe & 2-Hexenal & All \\
\hline & tLinox & trans-Linalool oxide & All \\
\hline & cLinox & cis-Linalool oxide & All \\
\hline & Lina & Linalool & All \\
\hline & aTerpol & $\alpha$-Terpineol & All \\
\hline & pMenth & p-Menth-1-en-9-al & All \\
\hline & bDam & $\beta$-Damascenone & All \\
\hline \multirow{31}{*}{ 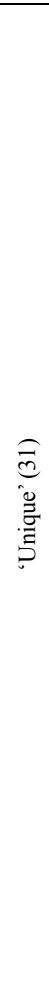 } & aTerp & $\alpha$-Terpinene & 'Pioneer' \\
\hline & U1 & Unidentified 1 & 'Pioneer' \\
\hline & Non & Nonanal & 'Sapphire' \\
\hline & Pin & $\alpha$-Pinene & 'Sapphire' \\
\hline & Cyclo & 1,3 , Cyclo-hexadiene $1,3,5,5$,tetra-methyl & 'Sapphire' \\
\hline & Iso & 2-Isopropylidene-5-methylhex-4-enal & 'Sapphire' \\
\hline & Eug & Eugenolmethylether & 'Sapphire' \\
\hline & Gera & cis-Geranylacetone & 'Sapphire' \\
\hline & $\mathrm{U} 2$ & Unidentified 2 & 'Laetitia' \\
\hline & Menth & Menthol & 'Laetitia' \\
\hline & U3 & Unidentified 3 & 'Laetitia' \\
\hline & $\mathrm{U} 4$ & Unidentified 4 & 'Laetitia' \\
\hline & U7 & Unidentified 7 & 'Songold' \\
\hline & U6 & Unidentified 6 & 'Songold' \\
\hline & cMenth & cis-p-Menth-2,8-dienol & 'Larry Anne' \\
\hline & U9 & Unidentified 9 & 'Larry Anne' \\
\hline & 2But & 2-[(2E)-2-Butenyl]-1,3,5-trimethylbenzene & 'Larry Anne' \\
\hline & U10 & Unidentified 10 & 'Larry Anne' \\
\hline & But & Butyl acetate & 'Flavor King' \\
\hline & U8 & Unidentified 8 & 'Flavor King' \\
\hline & Pent & Pentamethylene acetate & 'Flavor King' \\
\hline & 3 Hexe & cis-3-Hexenol & 'Flavor King' \\
\hline & Hexfor & n-Hexylformate & 'Flavor King' \\
\hline & Amyl & Amylacetate & 'Flavor King' \\
\hline & Oct & Octyl acetate & 'Flavor King' \\
\hline & Butbu & Butylbutanoate & 'Flavor King' \\
\hline & 3Hexac & cis-3-Hexenyl acetate & 'Flavor King' \\
\hline & Hep & Heptyl acetate & 'Flavor King' \\
\hline & Benzyl & Benzyl acetate & 'Angeleno' \\
\hline & U5 & Unidentified 5 & 'Angeleno' \\
\hline & Pyran2 & 2H-Pyran,2-ethenyltetrahydro-2,6,6tri-methyl & 'Angeleno' \\
\hline \multirow{23}{*}{ 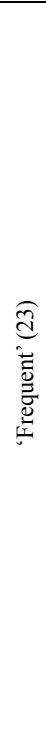 } & Limox & Limonene oxide & 'Pioneer', 'Larry Anne' \\
\hline & Cymol & p-Cymen-8-ol & 'Songold', 'Angeleno' \\
\hline & Phe & Phenol & 'Laetitia', 'Songold' \\
\hline & Nona & Nonanoic acid & 'Sapphire', 'Flavor King', \\
\hline & Bisa & Bisabalol oxide B & 'Laetitia', 'Angeleno' \\
\hline & Hex & 1-Hexanol & 'Laetitia', 'Songold', 'Angeleno' \\
\hline & Eth & 2-Ethylfuran & 'Laetitia', 'Songold', 'Angeleno' \\
\hline & Cym & $\beta$-Cymene & 'Pioneer', 'Songold', 'Larry Anne' \\
\hline & Lim & D-Limonene & 'Pioneer', 'Larry Anne', 'Angeleno' \\
\hline & Hexyl & n-Hexyl acetate & 'Sapphire', 'Flavor King', 'Larry Anne' \\
\hline & Deca & $\gamma$-Decalactone & 'Sapphire', 'Flavor King', 'Larry Anne' \\
\hline & Dodeca & $\gamma$-Dodecalactone & 'Sapphire', 'Flavor King', 'Larry Anne' \\
\hline & Trimeth6 & 4-(2,6,6-Trimethyl-cyclohexa-1,5,-diemethyl)but-3-en-2-one & 'Sapphire', 'Flavor King', 'Songold' \\
\hline & Car & 3-Carene & 'Pioneer', 'Sapphire,', 'Larry Anne', 'Angeleno' \\
\hline & Oci & Ocimenol & 'Sapphire', 'Flavor King', 'Larry Anne', 'Angeleno' \\
\hline & Trimeth & 4-(2,4,4-Trimetyl-cyclohexa-1,5-dienyl)but-3-en-2-one & 'Pioneer', 'Sapphire', 'Laetitia', 'Flavor King', 'Songold' \\
\hline & aIon & $\alpha$-Ionone & 'Pioneer', 'Songold', 'Flavor King', 'Larry Anne', 'Angeleno' \\
\hline & Benz & Benzaldehyde & 'Laetitia', 'Flavor King' Songold', 'Larry Anne' 'Angeleno' \\
\hline & Hexen & 2-Hexen-4-olide & 'Laetitia', 'Flavor King' Songold', 'Larry Anne' 'Angeleno' \\
\hline & Pyran3 & 2H-Pyran,3,6.dihydro-4-methyl-2-(2-methyl-1-propenyl) & 'Pioneer', 'Sapphire', 'Flavor King', 'Songold', 'Larry Anne' \\
\hline & Hot & Hotreinol & 'Pioneer', 'Sapphire', 'Laetitia', 'Flavor King', 'Larry Anne', 'Angeleno' \\
\hline & Bor & 2-Bornene & 'Pioneer', 'Sapphire', 'Laetitia', 'Flavor King', 'Larry Anne', 'Angeleno' \\
\hline & blon & $\beta$ - Ionone & 'Pioneer', 'Sapphire', 'Laetitia', 'Flavor King', 'Songold', 'Larry Anne' \\
\hline
\end{tabular}

Results for 'Pioneer', 'Laetitia' and 'Angeleno' are taken from Louw and Theron ${ }^{5}$. Abb = abbreviation 
Table 5. Variable selection table resulting from a stepwise DA of the three functional groups within each cultivar

\begin{tabular}{|c|c|c|c|c|c|}
\hline Cultivar & Variable IN/OUT & Status & Partial $\mathrm{R}^{2}$ & $\mathrm{~F}$ & $\operatorname{Pr}>F$ \\
\hline \multirow[t]{9}{*}{ 'Sapphire' } & Hexe & IN & 0.433 & 12.600 & $<0.0001$ \\
\hline & Deca & IN & 0.350 & 8.616 & 0.001 \\
\hline & Неха & IN & 0.322 & 7.364 & 0.002 \\
\hline & Lina & IN & 0.349 & 8.047 & 0.002 \\
\hline & Deca & OUT & 0.127 & 2.190 & 0.130 \\
\hline & Trimeth & IN & 0.455 & 12.518 & 0.000 \\
\hline & Hexyl & IN & 0.224 & 4.185 & 0.025 \\
\hline & Hot & IN & 0.197 & 3.425 & 0.047 \\
\hline & bIon & IN & 0.255 & 4.627 & 0.019 \\
\hline \multirow[t]{9}{*}{ 'Flavor King' } & Hexe & IN & 0.878 & 118.376 & $<0.0001$ \\
\hline & Pent & IN & 0.672 & 32.750 & $<0.0001$ \\
\hline & aIon & IN & 0.491 & 14.973 & $<0.0001$ \\
\hline & Hot & IN & 0.367 & 8.699 & 0.001 \\
\hline & Nona & IN & 0.269 & 5.345 & 0.011 \\
\hline & Trimeth & IN & 0.445 & 11.247 & 0.000 \\
\hline & 3 Hexe & IN & 0.311 & 6.099 & 0.007 \\
\hline & Butbu & IN & 0.370 & 7.637 & 0.002 \\
\hline & Hexfor & IN & 0.333 & 6.237 & 0.006 \\
\hline \multirow[t]{5}{*}{ 'Songold' } & Phe & IN & 0.544 & 19.665 & $<0.0001$ \\
\hline & bDam & IN & 0.589 & 22.966 & $<0.0001$ \\
\hline & pMenth & IN & 0.657 & 29.650 & $<0.0001$ \\
\hline & aIon & IN & 0.536 & 17.324 & $<0.0001$ \\
\hline & Pyran3 & IN & 0.274 & 5.475 & 0.010 \\
\hline \multirow[t]{9}{*}{ 'Larry Anne' } & Limox & IN & 0.869 & 109.615 & $<0.0001$ \\
\hline & Oci & IN & 0.496 & 15.752 & $<0.0001$ \\
\hline & U10 & IN & 0.504 & 15.771 & $<0.0001$ \\
\hline & Pyran3 & IN & 0.443 & 11.919 & 0.000 \\
\hline & Cym & IN & 0.216 & 4.003 & 0.029 \\
\hline & Deca & IN & 0.581 & 19.448 & $<0.0001$ \\
\hline & bIon & IN & 0.358 & 7.516 & 0.003 \\
\hline & Lina & IN & 0.311 & 5.875 & 0.008 \\
\hline & Hexe & IN & 0.283 & 4.927 & 0.016 \\
\hline
\end{tabular}

The apparent similarities expressed by the significance testing mentioned above were not confirmed in the DA. In fact, the three functional groups appeared to be distinct with $100 \%$ correctly assigned samplesand clearly separated groups (Figure 1(a)). The only similarity was seen in the variables chart where the compounds seem to be equally spread throughout the 4 quadrants, most with similar distances from the origin (Figure 1(b)). The stepwise DA characterised the divergence by selecting eight compounds as contributing most to the pattern (Table 5). The top two compounds (2-hexenal and $\gamma$-decalactone) were also hugely affected by storage. When the DA was repeated using only the eight compounds identified by the stepwise method the divergence tended to lessen only slightly and the percentage correctly assigned compounds dropped to $94.44 \%$ with two 'Tree-ripened' samples incorrectly assigned to the 'Harvest' group. However, the functional groups remained different from each other implying that the eight compounds were indeed contributing to the variance. 
(a)
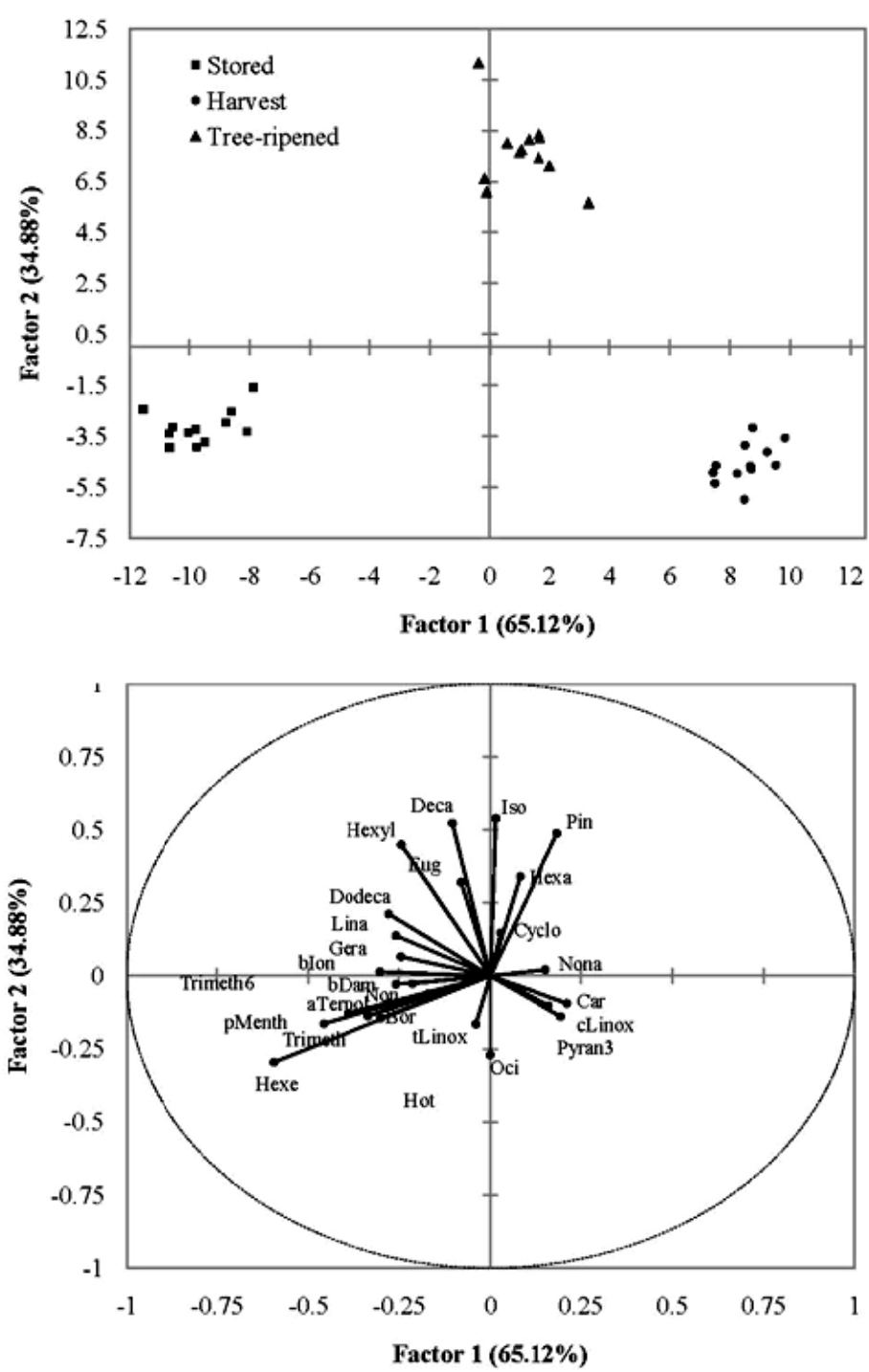

Figure 1. DA observations (a) and variables (b) charts for the functional groups of 'Sapphire'

\section{4 'Flavor King' Volatile Profile}

This plum $\mathrm{x}$ apricot hybrid had the most aroma volatiles of all the cultivars; a total of 32 compounds was detected with 10 uniquely associated with 'Flavor King' (Table 4). Twenty-nine compounds made up the volatile profile of the 'Harvest' group with cis-3-hexenol, octyl acetate and pentamethylene acetate not present at harvest (Table 3). Further characteristics of the 'Harvest' samples include significantly higher levels of hexanal, 2-hexenal, 2-hexen-4-olide, trans and cis linalool and hotrienol compared to the 'Tree-ripened' group and significantly lower levels of butyl acetate, n-hexylformate, heptyl acetate, $\mathrm{p}$-menth-1-en-9-al, $\beta$-ionone and the two $\gamma$-lactones (deca and dodeca) that seemed to be associated more with ripe fruit.Cold storage of 'Flavor King' fruit seemed to alter the volatile profile of the fruit considerably with a shift of almost $60 \%$ when compared to the profile of fruit ripened on the tree. No less than 19 components showed significant differences in their levels when exposed to cold storage with either increases in esters such as butyl acetate, amylacetate, butyl butanoate, cis-3-hexenyl acetateas well as cis-3-hexenol and hotrienol or decreases in hexanal, linalool, ocimenol, heptyl acetate, octyl acetate, the two ionones ( $\alpha$ and $\beta$ ) and the two $\gamma$-lactones (deca and dodeca) (Table 3). As these differences were shared between both 'generic' and 'unique' compounds suggesting that the general aroma and cultivar specific aroma changed during storage (Table 4). 
(a)
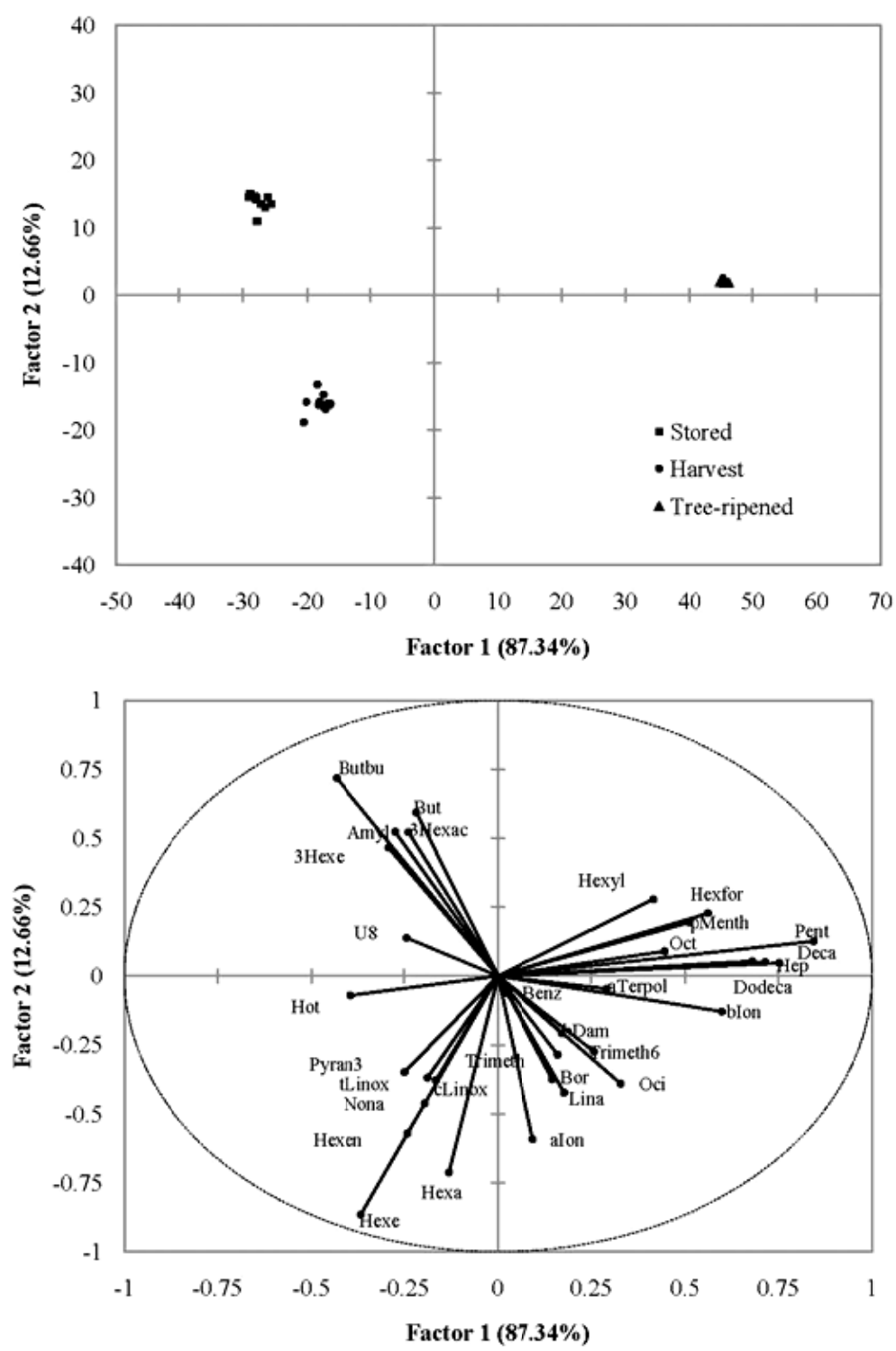

Figure 2. DA observations (a) and variables (b) charts for the functional groups of 'Flavor King'

The observation chart (Figure 2(a)) of the DA illustrated the differences in the three functional groups with large distances amongst the groups. When comparing the scale of the axes to those of the 'Sapphire' chart it became evident that the divergence amongst the functional groups of 'Flavor King' and their associated volatile profiles (Figure 2(b)) was much greater. The cause of this separation pattern was explained in more detail by the nine components identified by the stepwise analysis (Table 5). Hexenal, pentamethylene acetate and $\alpha$-ionone were selected as the top most compounds responsible for the differences in the volatile profiles. When reworking the DA using only the nine compounds from the stepwise list the functional groups remained distinct, but moved somewhat closer to each other, more similar compared to a true plum such as 'Sapphire'. Reclassification testing validated both the DA and stepwise DA with $100 \%$ correctly assigned samples.

\section{5 'Songold' Volatile Profile}

Similar to the early cultivar 'Pioneer' (Louw \& Theron, 2012) we recorded a total of 22 compounds in 'Songold' (Tables 3 and4). When compared to the cultivars analysed in the present study, six unique compounds were found, but when combined with the results from Louw and Theron (2012) the amount decreased to only two compounds that could not be identified without a significant amount of uncertainty (Table 4). All 22 compounds 
maintained their presence from the 'Harvest' to the 'Tree-ripened' phase, but during cold storage one component, p-cymen-8-ol, decreased to undetectable levels (Table 3). Following the pattern of 'Laetitia' and 'Pioneer' the 'Songold' 'Harvest' samples also had the majority of its compounds at their highest levels, including five of the 'generic' compounds (Louw \& Theron, 2012). The 'Tree-ripened' fruit showed rising levels of 1-hexanol, 2-ethylfuran, 2-hexen-4-olide and $\beta$-ionone whilst maintaining relatively high levels of the generic compounds $\beta$-damascenone, hexanal and 2-hexenal. Cold storage altered the ripening of the 'Songold' in such a way that nine of the 22 compounds in the 'Stored' functional groups were significantly different from the 'Tree-ripened' fruit. Most of the affected compounds decreased drastically during storage indicating failure to maintain or increase their levels except for 1-hexanol that accumulated levels of more than double compared to the 'Tree ripened' fruit and more than 52 times its harvest level. The compounds 4-(2,4,4-trimetyl-cyclohexa-1,5-dienyl)but-3-en-2-one and unidentified 6, however, managed to maintain their harvest levels during storage suggesting a failure to metabolise these compounds as seen in the 'Tree-ripened' samples or possibly a loss followed by a resynthesisation.
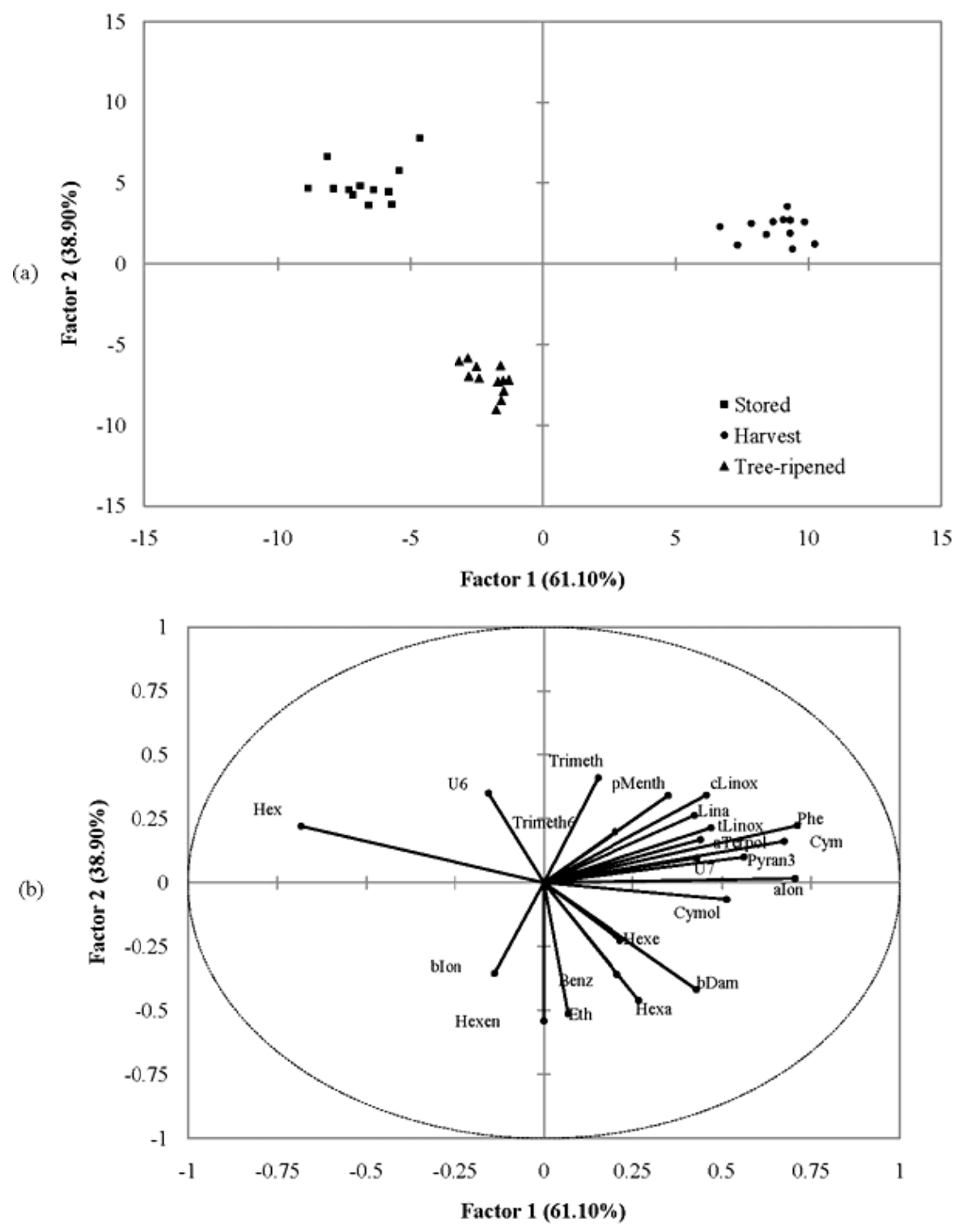

Figure 3. DA observations (a) and variables (b) charts for the functional groups of 'Songold'

As expected, the DA results of 'Songold' indicated three distinct functional groups (Figure 3(a)) with 100\% correctly assigned samples upon reclassification. The variables plot confirmed the volatile profiles described 
above (Figure 3(b)) with most compounds found in quadrant 1 similar to the 'Harvest' samples and specific compounds scattered in the third and fourth quadrants in correlation with the 'Tree-ripened' and 'Stored' groups. Stepwise DA selected five variables as the main compounds responsible for the separation of the groups (Table 5) with relatively high partial $\mathrm{R}^{2}$ values for the first four compounds. Interestingly, phenol that only occurred in the 'Harvest' fruit of 'Laetitia' topped the list and was again mostly associated with the 'Harvest' samples, although now present in all three groups (Louw \& Theron, 2012). The effects of these compounds are real as they continued to separate the functional groups when tested in isolation although the distances between the groups seem to be closer than before; reclassification remained at $100 \%$ correctly assigned samples.

\section{4 'Larry Anne' Volatile Profile}

(a)

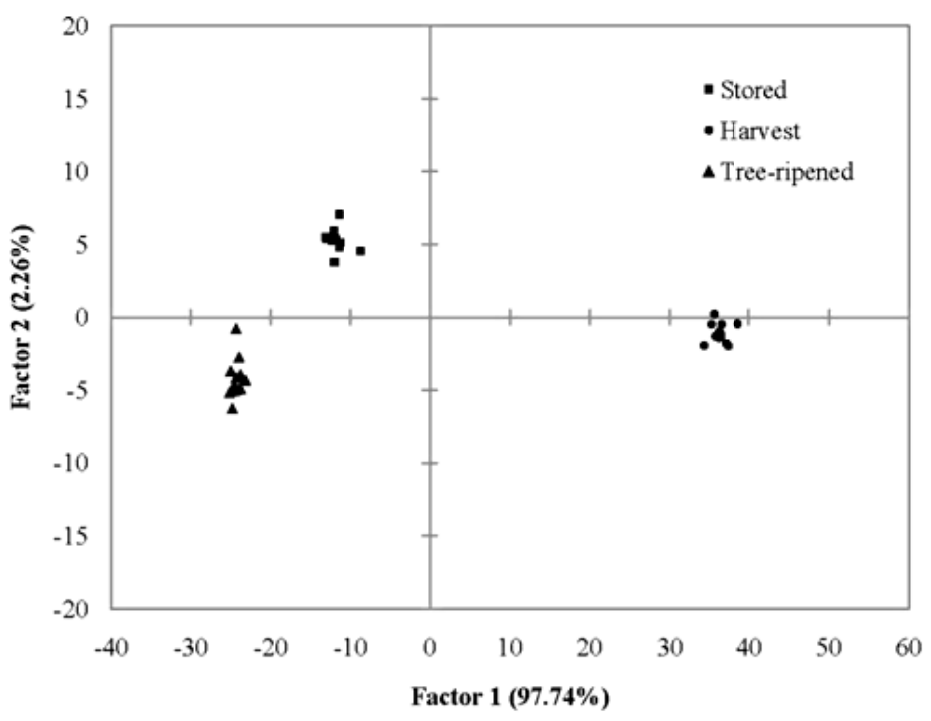

(b)

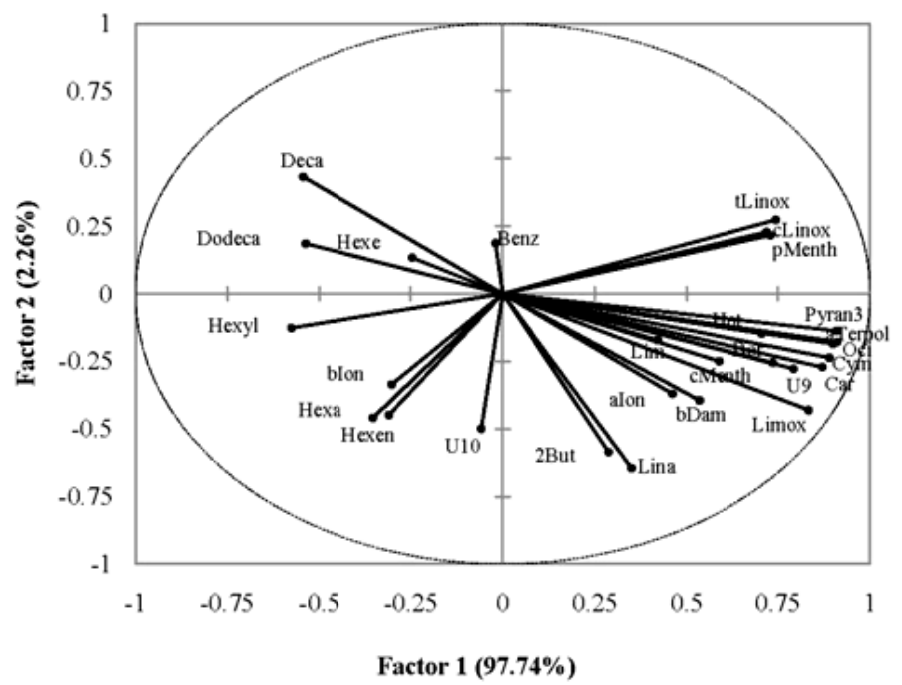

Figure 4. DA observations (a) and variables (b) charts for the functional groups of 'Larry Anne'

'Larry Anne' contained the most (27) aroma compounds of the true plum cultivars tested (Table 3 and 4). It represented all the 'generic' and four 'unique' compounds of which two (2-[(2E)-2-butenyl]-1,3,5-trimethylbenzene and cis-p-menth-2,8-dienol) were positively identified. In general, all the compounds were present in all of the functional stages with the exception of $n$-hexyl acetate being absent from the 'Harvest' samples. Again, as seen in most of the plum cultivars, the 'Harvest' samples contained the highest level of the majority of the compounds with decreasing trends during ripening. Significantly lower levels were 
only measured for hexanal and 2-hexen-4-olide and, similarly to 'Flavor King', low levels were also detected in the 'Harvest' samples for the two $\gamma$-lactones and $\beta$-ionone. These five compounds, together with $n$-hexyl acetate, accounted for the high level compounds found in the 'Tree-ripened' fruit. Also similar to 'Flavor King', cold storage seemed to have a confounding effect on the volatile profile of the fruit with $56 \%$ of the compounds altered when compared to fruit left to ripen on the tree. The general pattern caused by long term cold storage seemed to be the decreasing of compound levels to amounts significantly different to both the 'Harvest' and 'Tree-ripened' groups. 2-Hexen-4-olide, $\beta$-ionone, $\gamma$-dodecalactone and $n$-hexylacetate also followed this trend but did not dip below the levels detected in the 'Harvest' fruit.Five compounds (p-menth-1-en-9-al, 2-hexenal benzaldehyde and the two linalool oxides) showed levels much higher after storage when compared to 'Tree-ripened' fruit. Interestingly, hexanal and $\gamma$-decalactone seemed to be unaffected by cold storage and have accumulated to a similar level as found in the 'Tree-ripened' fruit.

In some aspects, the DA results for 'Larry Anne' seemed to be similar to those of 'Flavor King'. There was a large divide amongst the three functional groups, the values of the $\mathrm{x}$-axis were also much larger than those found in the other true plum cultivars (Figure 4(a)) and the variables chart was scattered with compounds a similar distance from the origin (Figure 4(b)). Nine compounds were identified by the stepwise DA (Table 5) with the top five compounds from the 'frequent' classification. No 'unique' compounds were on the list and only three 'generic' compounds were found at the bottom of the list. In the light of these results, it was expected that the confusion matrixes both showed $100 \%$ correctly assigned samples. When the nine compounds selected by the stepwise method were reanalysed in a DA the observations chart showed the functional groups now closer to one another and more similar to the patterns seen in the other cultivars, however, all the volatile profiles remained distinct with $100 \%$ correct reclassification.

\subsection{Comparing the Functional Groups of the Cultivars}

From the intra-cultivar results presented thus far it was evident that the functional groups within each cultivar had a different volatile profile. To compare the functional groups of the cultivars with one another the DA was repeated using the cultivars as the dependent variables.

From the observation plot for the 'Harvest' groups (Figure 5(a)) it was visually evident that 'Sapphire' 'Songold' were well separated from 'Flavor King' and 'Larry Anne'. This suggested that the volatile profiles of these two are perhaps more similar to each other at harvest than to 'Flavor King' and especially 'Larry Anne'. When compared to Figure 5(b) the volatile profiles were illustrated relative to the four cultivars with $99.99 \%$ of the variance described by Factors 1 and 2. The stepwise analysis identified only 6 of the 62 compounds as significant contributors to the pattern with limonene oxide and cis-3-hexenyl acetate, two 'frequent' compounds, at the top of the list (Table 6). Only one of the eight 'generic' compounds (linalool) appeared on the list and could possibly indicate that the general plum aroma of the four cultivars were similar at harvest. It should also be kept in mind that 'Harvest' fruits were believed to be physiologically mature although not yet ripe, and therefore, it is expected that the volatile profiles might still be under-developed. When the six stepwise-selected compounds were retested in isolation, the scale of the observation chart changed significantly moving the cultivars much closer together. The separation pattern, however, remained the same with 'Larry Anne' being far to the left. The confusion matrix remained at $100 \%$ implying that these compounds were indeed responsible for the divergent volatile profiles.

Once the fruit had ripened on the tree the DA showed a divergence pattern similar to that of 'Harvest' with a large scale on both axis but now 'Flavor King' was well separated from the rest (Figure 6(a)). This might be expected as 'Flavor King' is a plum $\mathrm{x}$ apricot hybrid and thus strongly influenced by an apricot volatile profile that separates it from the true plums when ripe. The variables chart (Figure 6(b)) also illustrated the difference in the 'Flavor King' profile by lumping all of the compounds unique to 'Flavor King' in the bottom part of the first quadrant similar to where the 'Flavor King' samples were nested. The pattern in the 'Tree-ripened' volatile profiles seemed to be governed by more compounds as for the 'Harvest' group with 20 different compounds selected by the stepwise analysis (Table 6). Interestingly, the list was crowned by cis-p-menth-2,8-dienol and amylacetate that belonged to the 'unique' collection of compounds for 'Larry Anne' and 'Flavor King' respectively. Although none of the 'generic' compounds were selected, the list was heavily populated with 'unique' compounds that aid in segregating each cultivar from the rest. Repeat analysis using on the 20 compounds selected by the stepwise DA showed an unchanged distribution of the four cultivars and a $100 \%$ correctly assigned samples upon reclassification. 
Table 6. Variable selection table resulting from a stepwise DA for the three functional groups across the four cultivars

\begin{tabular}{|c|c|c|c|c|c|}
\hline Functional group & Variable IN/OUT & Status & Partial $\mathrm{R}^{2}$ & $\mathrm{~F}$ & $\operatorname{Pr}>\mathrm{F}$ \\
\hline \multirow[t]{6}{*}{ Harvest } & Limox & IN & 0.977 & 610.304 & $<0.0001$ \\
\hline & 3Нехас & IN & 0.868 & 93.979 & $<0.0001$ \\
\hline & alon & IN & 0.608 & 21.678 & $<0.0001$ \\
\hline & 2But & IN & 0.593 & 19.871 & $<0.0001$ \\
\hline & Lina & IN & 0.430 & 10.063 & $<0.0001$ \\
\hline & Trimeth6 & IN & 0.351 & 7.033 & 0.001 \\
\hline \multirow[t]{24}{*}{ Tree-ripened } & cMenth & IN & 0.914 & 146.790 & $<0.0001$ \\
\hline & Amyl & IN & 0.797 & 56.215 & $<0.0001$ \\
\hline & Hex & IN & 0.613 & 22.146 & $<0.0001$ \\
\hline & U6 & IN & 0.584 & 19.202 & $<0.0001$ \\
\hline & Hexfor & IN & 0.579 & 18.322 & $<0.0001$ \\
\hline & Trimeth6 & IN & 0.359 & 7.274 & 0.001 \\
\hline & aIon & IN & 0.517 & 13.552 & $<0.0001$ \\
\hline & Eug & IN & 0.427 & 9.206 & 0.000 \\
\hline & Hot & IN & 0.351 & 6.486 & 0.001 \\
\hline & Pent & IN & 0.373 & 6.939 & 0.001 \\
\hline & 3Hexe & IN & 0.267 & 4.137 & 0.013 \\
\hline & 3Нехас & IN & 0.346 & 5.809 & 0.003 \\
\hline & U7 & IN & 0.399 & 7.074 & 0.001 \\
\hline & U6 & OUT & 0.144 & 1.790 & 0.169 \\
\hline & Hexyl & IN & 0.327 & 5.190 & 0.005 \\
\hline & Pent & OUT & 0.026 & 0.281 & 0.839 \\
\hline & U8 & IN & 0.324 & 5.124 & 0.005 \\
\hline & Hexfor & OUT & 0.070 & 0.798 & 0.504 \\
\hline & Cym & IN & 0.262 & 3.787 & 0.020 \\
\hline & Hep & IN & 0.245 & 3.358 & 0.031 \\
\hline & Benz & IN & 0.316 & 4.623 & 0.009 \\
\hline & Oct & IN & 0.284 & 3.828 & 0.020 \\
\hline & Hep & OUT & 0.105 & 1.129 & 0.353 \\
\hline & bIon & IN & 0.237 & 3.008 & 0.046 \\
\hline \multirow[t]{21}{*}{ Stored } & U9 & IN & 0.962 & 845.630 & $<0.0001$ \\
\hline & Phe & IN & 0.901 & 129.898 & $<0.0001$ \\
\hline & U8 & IN & 0.742 & 40.202 & $<0.0001$ \\
\hline & Hexen & IN & 0.533 & 15.604 & $<0.0001$ \\
\hline & Eth & IN & 0.390 & 8.540 & 0.000 \\
\hline & Hex & IN & 0.687 & 28.528 & $<0.0001$ \\
\hline & U10 & IN & 0.424 & 9.331 & $<0.0001$ \\
\hline & Limox & IN & 0.473 & 11.087 & $<0.0001$ \\
\hline & Cymol & IN & 0.568 & 15.798 & $<0.0001$ \\
\hline & cMenth & IN & 0.694 & 26.521 & $<0.0001$ \\
\hline & 2But & IN & 0.811 & 48.593 & $<0.0001$ \\
\hline & Сym & IN & 0.390 & 7.044 & 0.001 \\
\hline & U7 & IN & 0.404 & 7.242 & 0.001 \\
\hline & Limox & OUT & 0.116 & 1.406 & 0.259 \\
\hline & aIon & IN & 0.370 & 6.251 & 0.002 \\
\hline & Butbu & IN & 0.389 & 6.584 & 0.001 \\
\hline & Amyl & IN & 0.568 & 13.151 & $<0.0001$ \\
\hline & U6 & IN & 0.430 & 7.285 & 0.001 \\
\hline & U7 & OUT & 0.086 & 0.914 & 0.446 \\
\hline & Lim & IN & 0.475 & 8.745 & 0.000 \\
\hline & But & IN & 0.258 & 3.254 & 0.036 \\
\hline
\end{tabular}



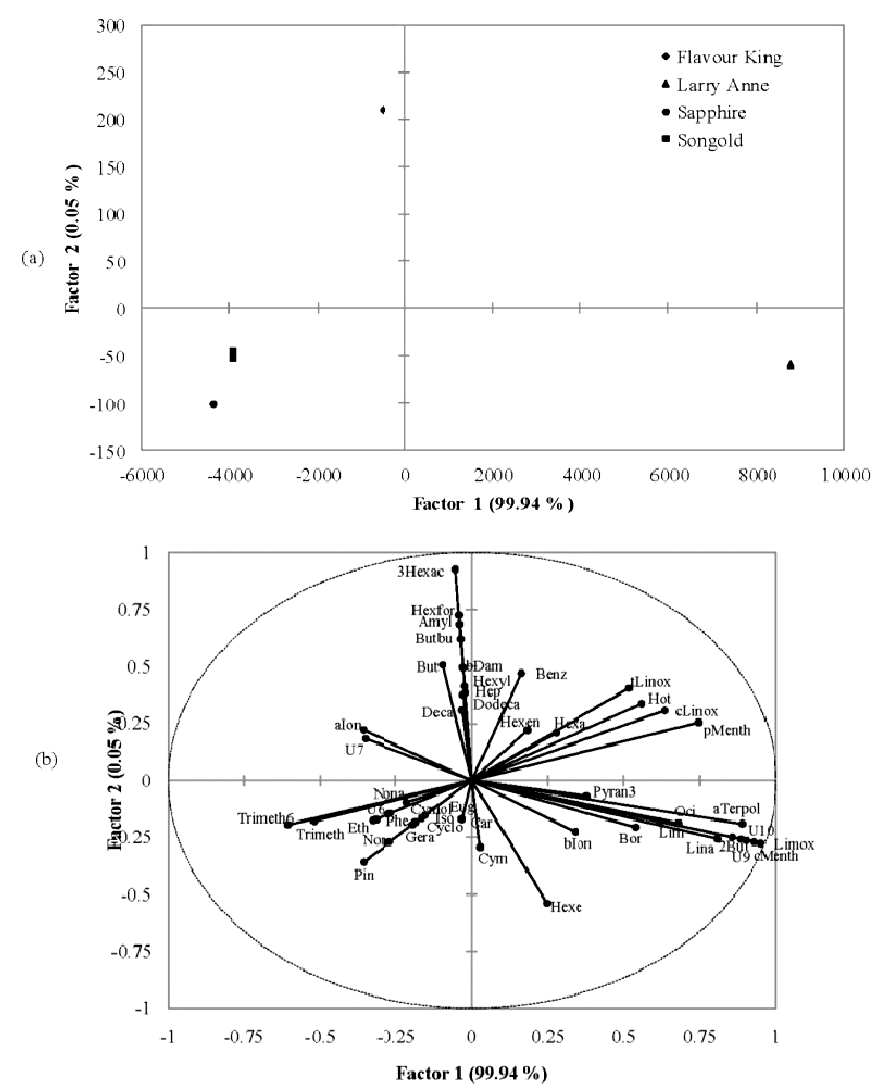

Figure 5. DA observations (a) and variables (b) charts for the 'Harvest' functional group

(a)
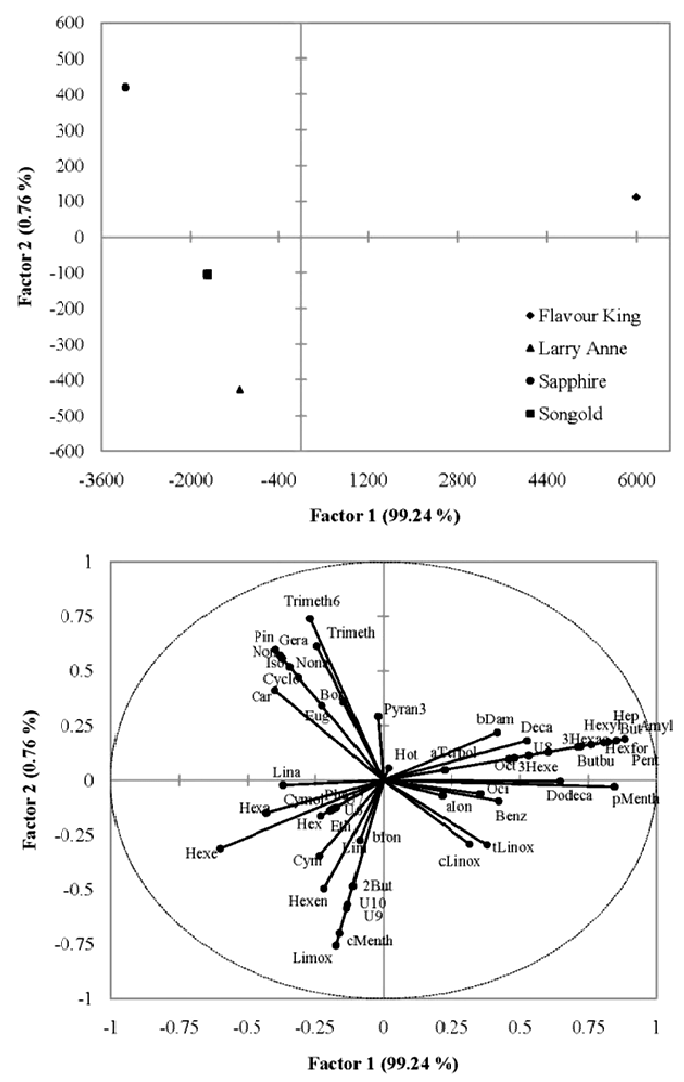

Figure 6. DA observations (a) and variables (b) charts for the 'Tree-ripened' functional group 
When the DA of the 'Stored' samples (Figure 7 and Table 6) was studied it appeared that the four cultivars maintained the pattern of separation seen in the 'Harvest' and 'Tree-ripened' group. Interestingly, 'Flavor King', and 'Larry Anne' had swapped positions and were now similar to what was seen in the 'Harvest' samples with 'Larry Anne' to the far right of the graph. The scale of the x-axis had noticeably increased indicating a further drift amongst the volatile profiles of the 'Stored' samples. Compared to the 'Tree-ripened' distribution pattern the biggest difference brought about by the low temperature exposure seemed to be in the positioning of 'Larry Anne' and 'Flavor King' relative to the rest of the cultivars. They had not just changed position, but also increased their distance from the other cultivars suggesting a large shift in their volatile profiles. This effect confirmed what was seen in the intra-cultivar analysis of these two cultivars where the apparent changes in the volatile profiles caused by cold storage were also most severe. The stepwise DA identified 19 of the 52 compounds as the main contributors to the observed pattern (Table 6) with again the 'unique' compounds, unidentified 9 and unidentified 8 , belonging to 'Larry Anne' and 'Flavor King' respectively, topping the list with phenol which in this study is unique to 'Songold'. Again, it is important to bear in mind that although this discussion labeled some cultivars as 'like' or 'similar' with respect to their volatile profiles, they all remained $100 \%$ distinct upon validation with no apparent overlap.
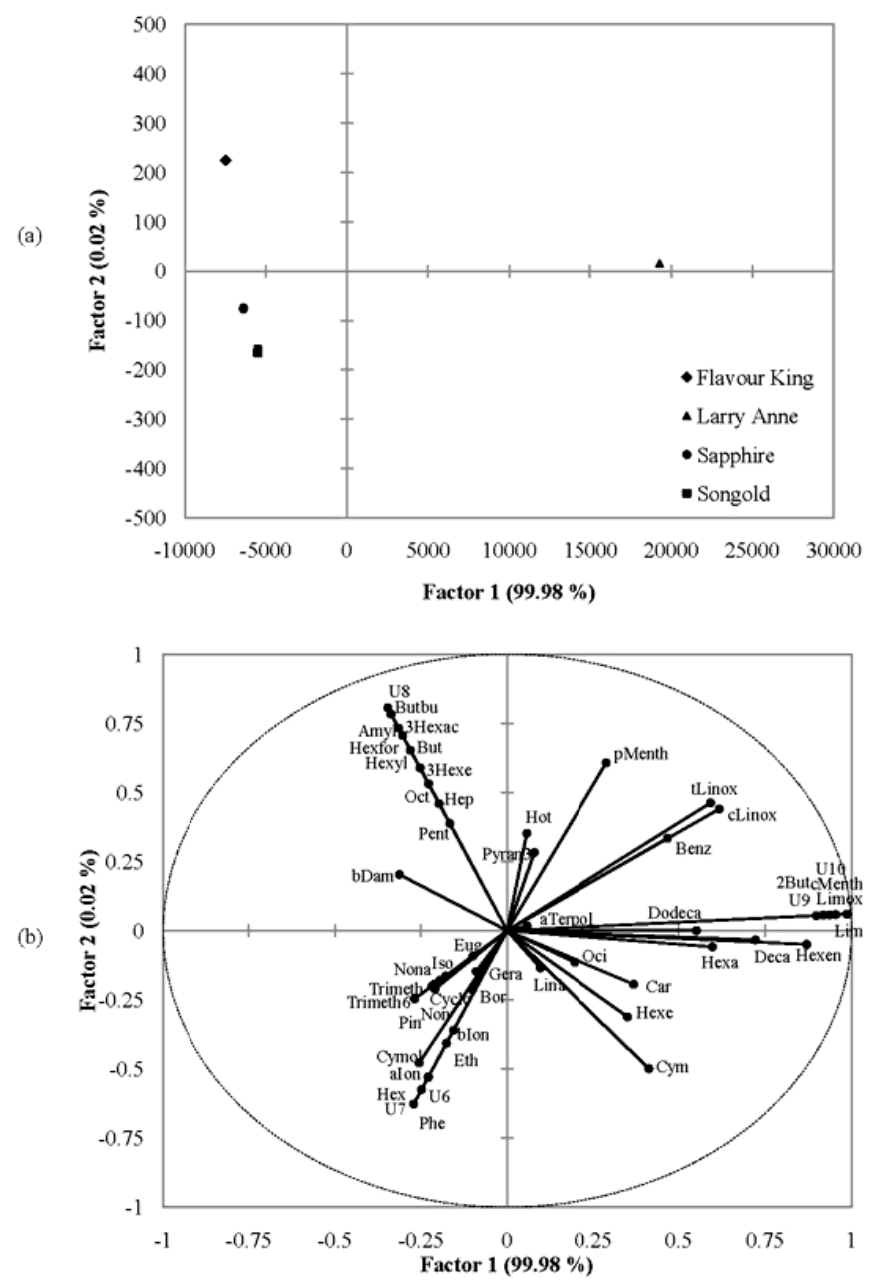

Figure 7. DA observations (a) and variables (b) charts for the 'Stored' functional group

\section{Discussion}

Cold storage extends the postharvest life of fruit by lowering the respiration and ethylene biosynthesis rates, thereby delaying ripening and senescence. Counterproductive to this, the lowering of respiration rates and ethylene production has a negative impact on the aroma quality of fruit by lowering the supply of precursors (especially fatty acid precursors) to the biochemical pathways leading to the formation of aroma compounds 
(Song \& Bangerth, 2003). The results above showed that the ripening of plums and the effects of cold storage seemed to be cultivar specific with unique profiles for each of the functional groups indicating a shift in the volatile composition as the fruit ripened and/or were exposed to long term cold storage conditions.

When considering the different chemical groups measured within the seven cultivars, there were some prominent compounds and general trends worth discussing even if there is no existing literature, apart from Louw and Theron (2012), on plum, storage studies from which to draw direct comparisons.

The alcohol group was mostly characterised by 1-hexanol although only present in 'Songold' samples with a significant increase in ripe fruit and showing a steep increase in stored samples. Contrary to this, in 'Angeleno' and 'Laetitia' samples it was strongly associated with 'Tree-ripened' fruit with significantly higher levels that were not maintained nor increased during storage (Louw \& Theron, 2012). 1-Hexanol is commonly described as having a 'green' aroma and has been widely identified in ripe European and Japanese plums and plumcots (Ismail, Williams, \& Tucknott, 1981a; Gómez \& Ledbetter, 1994; Gómez \& Ledbetter, 1997). Interestingly it has been recorded to be present at a lower level in ripe plums with a strong plum-like odour and is said to have a negative, overpowering effect on compounds imparting plum-like odours (Ismail et al., 1981a).

The aldehydes, hexanal and 2-hexenal, were prominent in all the cultivars measured in the present study and that of Louw and Theron (2012) and were usually present in relatively high values at harvest and throughout ripening.Ismail, Williams andTucknott (1981b) and Guichard, SchlickandIssanchou (1990) describes these compounds as having a strong 'green' aroma associated with immature fruit and diminishing in concentration as ripening proceeds rather than remaining relatively constant as found in this study (Gómez \& Ledbetter, 1997). Some plum aroma studies also identified these compounds in relatively high amounts in ripe plums (Ismail et al., 1981a; Gómez \& Ledbetter, 1994; Ismail et al., 1981b). Etievant, Guichard and Issanchou (1986) reported that the levels of both these aldehydes increased after deep-freezing and thawing of plum samples implying that their values can thus be inflated if samples have been subjected to such procedures prior to assessment. Raffo et al. (2008) also warned against massive formation of these $\mathrm{C}_{6}$-aldehydes originating from increased lipoxygenase activity associated with the crushing of fruit and suggested an enzyme deactivation step with saturated $\left(\mathrm{NH}_{4}\right)_{2} \mathrm{SO}_{4}$ during sample homogenisation to prevent inflated aldehyde levels in the results. In spite of such efforts Raffo et al. (2008) still found that these compounds represented the main fraction of the whole volatile compounds isolated from peach samples. Similar to our storage results Raffo et al. (2008) reported that they did not observe univocal effects of cold storage on hexanal and 2-hexenal in two peach cultivars after two weeks at $1{ }^{\circ} \mathrm{C}$. In the light of the high presence and apparent importance of these compounds, it is not surprising that they were both often identified via the stepwise DA as important contributors to the separation patterns of intra-cultivar functional groups. Another aldehyde worth mentioning is p-menth-1-en-9-al, which was measured in relatively high amounts in all four plum cultivars in the present study similar to that reported by Louw and Theron (2012), but which has not been reported in any plums or stone fruit related studies. It is, however, commonly found in citrus honey (Soria, Sanz, \& Martìnez-Castro, 2009). Cold storage caused p-menth-1-en-9-al levels to increase and decrease in some cultivars but four out of the seven showed no significant differences associated with low temperature exposure.

Esters (together with alcohols and aldehydes) are reported to form the most numerous chemical substances identified in plum extracts, contribute to the fruity aroma in plums and some can act as a molecular tracer of apricot aromatic quality (Gómez \& Ledbetter, 1994; Crouzet, Etievant, \& Bayonove, 1990; Guillot et al., 2002). In contrast to this we found esters (nine in total) to be limited to mainly the plumcot 'Flavour King' with only n-hexal acetate and benzyl acetate measured in two other true plum species. Gómezand Ledbetter (1997) also reported the presence of numerous esters during the ripening of a plumcot accession, 'P251-002', with three being significant to the volatile profile, although they did not appear in either parent cultivars (Gómez \& Ledbetter, 1993). In a recent study comparing the volatile profiles of six Japanese plums Lozano et al. (2009) reported $n$-hexyl acetate as one of the esters presents in the greatest proportions with similar levels detected in ripe 'Larry Anne' and 'Songold' and lower levels in 'Angeleno' ('Suplumsix'). We failed to detect n-hexyl acetate in any of our 'Songold' (present study) or 'Angeleno' samples, however, the levels found in 'Larry Anne', Sapphire' and 'Flavor King' had strong links to ripe plum samples and seemed to develop during the ripening process as the mature but unripe fruit in the 'Harvest' group had significantly lower levels (Louw \& Theron, 2012). Cold storage had an inhibiting effect on the accumulation of n-hexyl acetate and resulted in samples with levels lower than the 'Tree-'ripened' but higher than the 'Harvest' fruit. Butyl acetate, although only measured in 'Flavour King', reached a level almost three times that found in tree-ripened fruit and almost 19 times that found in harvest fruit. This significant increase suggested that low temperatures can favour the formation and accumulation of this specific ester. Butyl acetate was reported in other tree-ripened Japanese plum cultivars 
(Gómez \& Ledbetter, 1994). However, Lozano et al. (2009), similar to the present findings, also failed to quantify any levels for ripe 'Songold', 'Larry Anne' and 'Angeleno' ('Suplumsix') samples.

For the furan chemical group, we could not find univocal patterns amongst the compounds although 2-hexen-4-olide seemed to be associated mostly with harvest fruit as some cultivars had higher levels compared to ripe and stored samples. Even the 'generic' compounds trans- andcis- linalool oxides showed ambiguous trends during ripening and storage for the cultivars in the present study.

The hydrocarbon compounds appeared equally uneventful except for relatively high levels of hotrienol recorded in six of the seven cultivars when combining results from Louw and Theron (2012). Only 'Flavour King' was sensitive to cold storage effects with hotrienol levels in the 'Stored' functional group remaining at a level equal to that of the 'Harvest' group and higher than that of the 'Tree-ripened' group indicating that prolonged exposure to low temperatures interrupts its metabolism. Hotrienol was not identified in any other plum or stone fruit cultivars but is commonly found in other fruit types such as nectarines (Engel et al., 1988), grapes (Williams, Strauss, Wilson, \& Massy-Westropp, 1982), passion fruit (Engel \& Tressl, 1983) and papaya (Schreier, Lehr, Heidlas, \& Idstein, 1985) as well as in citrus honey (Soria et al., 2009).

The lactones detected in 'Larry Anne', Sapphire' and 'Flavour King' are important as they have been identified in previous plum studies and are suggested to be indicative of ripeness as they increase during the ripening process (Williams \& Ismail, 1981; Gómez \& Ledbetter, 1994; Gómez \& Ledbetter, 1997). Williams and Ismail (1981) also suggested that $\gamma$-decalactone is responsible for creating a plum-like odour. In the present study, the levels of the two lactones, $\gamma$-decalatone and $\gamma$-dodecalactone, both increased significantly as the fruit ripened on the tree but in the case of $\gamma$-dodecalactone in 'Stored' fruit significantly lower levels were measured. The effect of cold-storage on the $\gamma$-decalactone levels seemed to be cultivar specific with increasing, decreasing and stable levels detected in different cultivars. In a cold storage study done on peaches lactone levels increased after one week of storage at $1{ }^{\circ} \mathrm{C}$ but drastically decreased after two weeks of storage under similar conditions, implying that the ability of the fruit to perform lactone accumulation and, consequently, to develop its aroma is reduced during prolonged cold storage (Raffo et al., 2008).

Norisoprenoids are volatile $\mathrm{C}_{9}-\mathrm{C}_{13}$ fragments from the degradation of $\mathrm{C}_{40}$-carotenoids, which have extremely low aroma thresholds (Mahatanatawee, Rouseff, Valim, \& Naim, 2005). Carotenoids are widely found in the plant kingdom and many fruit types have been documented to contain norisoprenoids, including stone fruit such as peaches (Raffo et al., 2008), apricots (Crouzet et al, 1990; Guillot et al., 2002) and plums (Williams \& Ismail, 1981; Gómez \& Ledbetter, 1994). The most prominent norisoprenoid measured in the present study was $\beta$-damascenone with high levels in both the 'Harvest' and 'Tree-ripened' fruit.It has also been described as having a camphor-like fruity aroma associated with the flavour of cooked plums (Williams \& Ismail, 1981). $\beta$-Damascenone levels seemed to be unaffected by cold storage and maintained its relatively high levels except for 'Songold' and 'Larry Anne' that showed a significant drop after storage. The other two norisoprenoids, $\alpha$ and $\beta$ ionones, showed similar trends with diminished levels after cold storage. Although Raffo et al. (2008) did not identify any of the three norisoprenoids mentioned above in their study on cold storage effects on peaches; they did find a decrease in the total amount of norisoprenoids in white-fleshed peaches after exposure to low temperature storage conditions.

In the terpenes group linalool and $\alpha$-terpineol are the most prominent. Both have been shown to have higher levels in the "Mature green" stage compared to the "Commercial ripe" and "Tree ripe" stages as identified for apricots and plumcots by Gómezand Ledbetter (1993). In the present study, no univocal pattern could be established for the levels of either of the two terpenes suggesting a cultivar-specific reaction to ripening and cold storage. Linalool has a floral woody aroma and a sensory evaluation done by Williams and Ismail (1981) found it to be repeatedly associated with a plum-like odour region of the chromatogram.

\section{Conclusion}

In conclusion, it is difficult to establish a general pattern to describe the reaction of plum volatiles to prolonged cold storage as each cultivar has a unique and complex profile already established at the unripe harvest stage, that diverges even further during tree-ripening and storage. It is, however, clear that some cultivars such as 'Flavor King' and 'Larry Anne' are more sensitive to cold storage and develop volatile profiles that are even more different from their 'Tree-ripened' profiles when compared to the other cultivars. From the results, it was also evident that the current commercial storage regimes, although favourable for quality parameters such as firmness, colour and sugar levels, altered the aroma profile of plums in such a way that it was neither 'Harvest'-like nor 'Tree-ripened'-like and delivered an end product with a different volatile quality. To overcome this it is suggested that the present study is expanded to include revised storage regimes and/or postharvest manipulations aimed at 
bridging the gap between the now diverged profiles of 'Stored' and 'Tree-ripened' plums. This should also be linked to consumer acceptance studies to determine the preferred volatile profiles and eating quality of export plums.

\section{Acknowledgements}

This study was sponsored by a research grant from SASPA (South African Stone Fruit Producers' Association).

\section{References}

Agozzino, P., Avellone, G., Filizzola, F., Farina, A., \& Lo Bianco, R. (2007). Changes in quality parameters and volatile aroma compounds in 'Fairtime' peach during fruit development and ripening. Italian Journal of Food Science, 1(19), 3-13.

Aubert, C., Bony, P., Chalot, G., \& Hero, V. (2010). Changes in physiochemical characteristics and volatile compounds of apricot (Prunus armeniaca L. cv. Bergeron) during storage and post-harvest maturation. Food Chemistry, 119, 1386-1398.http://dx.doi.org/10.1016/j.foodchem.2009.09.018

Blažek, J. (2007). A survey of the genetic resources used in plum breeding. ActaHorticulturae, 734, 31-45.

Crouzet, J., Etievant, P., \& Bayonove, C. (1990). Stoned fruit: apricot, plum, peach, cherry. In I. D. Morton \& A. J. Macloud (Eds.), Food Flavours Part C. The flavour of fruit. New York: Elsievier Science Publishing Company INC.

Engel, K. H., \& Tressl, R. (1983).Formation of aroma components from nonvolatile precursors in passion fruit. Journal of Agricultural and Food Chemistry,31, 998-1002.http://dx.doi.org/10.1021/jf00119a019

Engel, K. H., Flath, R. A., Buttery, R. G., Mon, T. R., Ramming, D. W., \& Teranishi, R. (1988). Investigation of volatile constituents in nectarines. 1. Analytical and sensory characterization of aroma components in some nectarine cultivars. Journal of Agricultural and Food Chemistry,36, 549-553. http://dx.doi.org/10.1021/jf00081a036

Etievant, P. X., Guichard, E. A., \& Issanchou, S. N. (1986). The flavour components of Mirabelle plums: Examination of the aroma constituents of fresh fruit; variation of head-space composition induced by deep-freezing and thawing. Sciences des Aliments, 6, 417-432.

Guichard, E., Schlick, P., \& Issanchou, S. N. (1990). Composition of apricot aroma: correlations between sensory and instrumental data. Journal of Food Science, 55, 735-738. http://dx.doi.org/10.1111/j.1365-2621.1990.tb05218.x

Guillot, S., Boulanger, R., Crouzet, J., Bureau, S., Lepoutre, J. P., \& Galindo, S. (2002). Tracers of apricot aromatic quality. The $10^{\text {th }}$ Weurmanflavour research symposium, Beaune, France.

Gómez, E., \& Ledbetter, C. A. (1993). Transmission of biochemical flavor constituents from apricot and plum to $\begin{array}{lllll}\text { their interspecific } \text { hybrids. Plant } & \text { 236-241. }\end{array}$ http://dx.doi.org/10.1111/j.1439-0523.1993.tb00634.x

Gómez, E., \& Ledbetter, C. A. (1994). Comparative study of the aromatic profiles of two different plum species: Prunus salicina Lindl and Prunus simonii L. Journal of the Science of Food and Agriculture, 65, 111-115. http://dx.doi.org/10.1002/jsfa.2740650116

Gómez, E., \& Ledbetter, C. A. (1997). Development of volatiles compounds during fruit maturation: Characterization of apricot and plum x apricot hybrids. Journal of the Science of Food and Agriculture, 74, 541-546.http://dx.doi.org/10.1002/(SICI)1097-0010(199708)74:4<541::AID-JSFA851>3.0.CO;2-D

Ismail, H. M., Williams, A. A., \& Tucknott, O. G. (1981a). The flavour components of plums: An examination of the aroma components present in the headspace above four cultivars of intact plums, Marjorie's Seedling, Merton Gem, NA 10, and Victoria. Journal of the Science of Food and Agriculture, 32, 498-502. http://dx.doi.org/10.1002/jsfa.2740320513

Ismail, H. M., Williams, A. A., \& Tucknott, O. G. (1981b). The favour of plums (Prunus domestica L.): An examination of the aroma components of plums from the cultivar Victoria. Journal of the Science of Food and Agriculture, 32, 613-619. http://dx.doi.org/10.1002/jsfa.2740320614

Louw, E. D., \& Theron, K. I. (2012). Volatile dynamics during maturation, ripening and cold storage of three Japanese plum cultivars (Prunus salicina Lindl.). Postharvest Biology and Technology, 70, 13-24. http://dx.doi.org/10.1016/j.postharvbio.2012.03.007

Lozano, M., Videl-Aragón, M. C., Hernández, M. T., Ayuso, M. C.,Bernalte, M. J., García, J., \& Velardo, B. 
(2009).Physiochemical and nutritional properties and volatile constituents of six Japanese plum (Prunus salicina Lindl.) cultivars. European Food Research and Technology, 228, 403-410. http://dx.doi.org/10.1007/s00217-008-0946-3

Mahatanatawee, K., Rouseff, R., Valim, M. F., \& Naim, M. (2005). Identification and aroma impact of norisoprenoids in orange juice. Journal of Agricultural and Food Chemistry, 53, 393-397. http://dx.doi.org/10.1021/jf049012k

PPECB (Perishable Products Export Control Board) Information portal, Cape Town, South Africa. Retrived from http://info.ppecb.com

Raffo, A., Nardo, N., Tabilio, M. R., \& Paoletti, F. (2008). Effects of cold storage on aroma compounds on white-and yellow-fleshed peaches. European Food Research and Technology, 226, 1503-1512.http://dx.doi.org/10.1007/s00217-007-0682-0

Schreier, P., Lehr, M., Heidlas, J., \& Idstein, H. (1985). Über das Aroma der Papayafrucht (Carica papaya, L.)Hinweise auf Vorstufen flüchtiger Verbindungen. Zeitschrift furLebensmittel-Untersuchung und Forschung, 180, 297-302.http://dx.doi.org/10.1007/BF01851273

Song, J., \& Bangerth, F. (2003). Fatty acids as precursors for volatile biosynthesis in pre-climacteric and climacteric apple fruit. Postharvest Biology and Technology, 30, 113-121.http://dx.doi.org/10.1016/S0925-5214(03)00098-X

Soria, A. C., Sanz, J., \& Martínez-Castro, I. (2009). SPME followed by GC-MS: a powerful technique for qualitative analysis of honey volatiles. European Food Research and Technology, 228, 579-590. http://dx.doi.org/10.1007/s00217-008-0966-z

Williams, A. A., \& Ismail, H. M. M. (1981). The volatile flavour components of plums and their sensory evaluation. In J. Solms \& R. Hall (Eds.), Criteria of Food Acceptance. Zurich: Forster publishing.

Williams, P. J., Strauss, C. R., Wilson, B., \& Massy-Westropp, R. A. (1982). Use of $C_{18}$ reverse-phase liquid chromatography for the isolation of monoterpene glycosides and nor-isopremoid precursors from grape juice and wines. Journal of Chromatography, 235, 471-480. http://dx.doi.org/10.1016/S0021-9673(00)85911-7 